\title{
Internet of Mobile Things: Overview of LoRaWAN, DASH7, and NB-IoT in LPWANs standards and Supported Mobility
}

\author{
Wael Ayoub $^{* \dagger}$, Abed Ellatif Samhat ${ }^{\dagger}$, Fabienne Nouvel*, Mohamad Mroue ${ }^{\dagger}$ and Jean-christophe Prévotet ${ }^{*}$ \\ ${ }^{*}$ Institut National des Sciences Appliquées de Rennes - IETR-INSA, Rennes, France. \\ ${ }^{\dagger}$ Faculty of Engineering - CRSI, Lebanese University, Hadath Campus, Hadath, Lebanon \\ Email*: firstname.lastname@insa-rennes.fr \\ Email ${ }^{\dagger}$ : samhat@ul.edu.lb, mohamad.mroue@ul.edu.lb
}

\begin{abstract}
Low-power Wide Area Networks (LPWANs) constitute a type of networks which is used to connect things to the Internet from a wide variety of sectors. These types of technologies provide the Internet of Things (IoT) devices with the ability to transmit few bytes of data for long ranges, taking into consideration minimum power consumption. In parallel, IoT applications will cover a wide range of human and life needs from smart environments (cities, home, transportation etc.) to health and quality of life. Among these popular LPWANs technologies, we have identified the unlicensed frequency band (LoRa, DASH7, SigFox, Wi-SUN, etc.), and the licensed frequency band standards (NB-IoT, LTE Cat-M, EC-GSM-IoT, etc.). In general, both types of standards only consider fixed interconnected things, and less attention has been provided to the mobility of the things or devices. In this paper, we address the mobility of the things and the connectivity in each of the three LPWAN standards: LoRaWAN, DASH7, and NB-IoT. In particular, we show how the mobility of things can be achieved when transmitting and receiving data. Then, we provide a general and technical comparison for the three standards. Finally, we illustrate several application scenarios where the mobility is required, and we show how to select the most suited standard. We also discuss the research challenges and perspectives.
\end{abstract}

Index Terms-IoT communication, LPWAN, LoRaWAN, DASH7, NB-IoT, Long-Range, Mobility.

\section{INTRODUCTION}

Throughout the last few years, Internet of Things (IoT) has attracted the attention of both industry and research communities in particular with the rise of Low Power Wide Area Networks (LPWANs) [1-3]. The IoT is sensitive to sustainable development [4] that will form a smart and comfortable future. IoT promises an interconnected network of smart things or objects including sensors, cameras, consumer electronic devices, etc. By 2020, there will be over billions of smart things connected to the Internet with a high potential economic impact, according to Cisco's expectation [5]. This will enable the integration of the software agents on the Internet and will make the interaction of the real world and the virtual world possible [6]. Adding to that, in [7], they predict that there will be more than 20.8 billion of smart things connected to the Internet by 2020 , whereas the worldwide number of connected devices was 6.4 billion in 2016. This growth is expected to continue to be exponential over the next decade, which introduces a rise in "Big Data" [8], energy consumption [9] and devices per cell [10]. Today, a wide range of data acquisition devices is already implemented in IoT applications
[11], [12], that require mobility such as smart cities [13-15], health-care [16], [17], smart vehicles [18], aging society [19], hospital [20], [21], and in post-emergency networks [22]. In such applications, the requirements for mobility, low latency, and long-range communication are significantly considerable [23]. For that, we will distinguish applications that require mobility and dynamic change of location from the rest of IoT applications with the term Internet of Mobile Things (IoMT) [24].

LPWANs describe a category of wireless communication technologies designed to support IoT deployments. LPWANs represent a new phenomenal model in communication that complements between cellular and short wireless technologies to address the diversity of IoT applications. These technologies are designed to offer a set of features including wide-area and massive scale connectivity [25] for low power, low cost, and low data rate devices.

One of the emerging protocols in this scope is the Long Range Wide-Area Network (LoRaWAN). LoRaWAN is one of the most popular and successful technologies in the LPWANs space. LoRaWAN consists of a protocol stack specified by LoRa Alliance [26] that operates over the Long Range (LoRa) physical layer on unlicensed bands. The LoRaWAN features are low data rate, low complexity, different operating classes for various applications. It may exhibit an immense number of nodes per single gateway. In 2015, LoRaWAN v1.0 was declared by LoRa Alliance. In October 2017, LoRa Alliance announced LoRaWAN v1.1.

Another well-defined LPWAN standard is the DASH7 Alliance Protocol (D7AP). D7AP is an open source Wireless Sensor and Actuator Network protocol (WSAN). It operates in the Sub-1 GHz bands based on the ISO/IEC 18000-7 standard and specified by DASH7 Alliance. The ISO/IEC 18000-7 standard defines the parameters of the active air interface communication at $433 \mathrm{MHz}$. D7AP inherits the default parameters [27] from ISO 18000-7 and extends the standard by specifying a complete communication stack from the application layer to the physical layer. This stack contains a high level of functionality optimized for active RFID and WSAN. Also, it ensures interoperability among different operators. Conversely to legacy RFID systems [28], D7AP supports tag-to-tag communication. In 2013, the D7AP 0.2 was announced by the DASH7 Alliance. In April 2016, the 
DASH7 Alliance published D7AP 1.1 [29].

Regarding cellular systems, there are several LTE releases [30], [31] focusing on low-power wide-area IoT connectivity. In Rel-12, LTE introduces low-cost devices comparable to GPRS [32], [33]. Pacing to support narrow-band machine to machine communications (MTC), LTE has introduced some key features in Rel-13. EC-GSM-IoT [34] and LTE-MTC [35] aim to enhance existing GSM and LTE networks. Their aim is to support "Rich IoT nodes and gateways" and "Mainstream" IoT applications. These types of applications are out of the scope of this paper and we only consider "constrained" IoT applications. Narrowband IoT (NB-IoT) [36] is based on existing LTE functionalities. This standard is optimized to achieve lowcost, ultra-low complexity, and indoor improvement coverage. It supports a huge number of devices per cell-site sector, low-power consumption, low-data-rate, and latency less than 10 seconds. NB-IoT has been developed to operate in three modes: in-band, guard-band, and stand-alone.

Whereas LoRaWAN and DASH7 use unlicensed frequencies that are globally available, NB-IoT uses the same frequencies as LTE which is implemented worldwide. Those standards are developed to satisfy the needs of constrained IoT communication requirements. However, they almost consider static interconnected things and pay less attention to the mobility of things.

In this paper, we present an overview of the three LPWAN standards: LoRaWAN, DASH7, and NB-IoT including architectures, specifications, and communications. Also, we provide a general and technical comparison between the three standards regarding deployment, coverage, cost, QoS, battery life, latency, and mobility. Motivated by the mobility and the connectivity requirements, we investigate in particular the three different LPWAN technologies regarding mobility support. Thus, we show how mobility could be achieved and describe the encountered limitations. Then, we illustrate several application scenarios and determine how to select the most suited standard.

The rest of this paper is organized as follows. Sections II, III, and IV give an overview of LoRaWAN, DASH7, and NB-IoT respectively and provide the necessary background information that will be intended to address mobility. In Section V, we focus on the mobility achievement of the movable things in both cases, when transmitting or receiving data. In section VI, we present a comparative study of the investigated standards regarding deployment, coverage, QoS, etc. Finally, Section VII provides a conclusion and discusses the future works.

\begin{tabular}{|c|c|c|}
\hline \multicolumn{3}{|c|}{ LoRaWAN (Data Link Layer) } \\
\hline Class A & Class B & Class C \\
\hline \multicolumn{3}{|c|}{ LoRa (Physical Layer) } \\
\hline
\end{tabular}

Fig. 1. LoRaWAN Protocol Architecture
TABLE I

LORA PROTOCOL SPECIFICATIONS

\begin{tabular}{|l|c|}
\hline Specification & LoRa Technology Support \\
\hline Standard & LoRa Alliance \\
\hline Operational Frequencies & Unlicensed ISM band 868, 915 MHz \\
\hline Modulation & Chirp spread spectrum (CSS) \\
\hline Coverage Range (Km) & $2-5$ (urban) $/ 15($ rural) \\
\hline Data Rate (kbps) & $0.3-50($ EU) $/ 0.9-100($ US) \\
\hline Topology & Star \\
\hline
\end{tabular}

\section{LORAWAN IOT TECHNOLOGY}

LoRaWAN is an open standard architecture developed by LoRa Alliance [26] to provide a medium access control mechanism and enable End-Devices (ED) to communicate with one or more gateways. LoRa is a physical layer technology that enables long range, low data rate, and low power wireless communication. It is an unlicensed band technology that modulates the signals in the sub GHz ISM band using the spread spectrum technique [37]. It was developed by Cycleo [38] and commercialized by Semtech [39], Microchip, and others. LoRa can also be applied in P2P communications between nodes. Table I shows the specifications of the LoRa protocol. LoRaWAN constitutes a data link layer protocol above the LoRa physical layer protocol as shown in Figure 1.

\section{A. Architecture}

LoRaWAN Alliance uses a star network topology, in which a gateway seamlessly relays messages between a Network Server (NS) and ED as shown in Figure 2. EDs use LoRa to communicate with Gateways (GW). GWs use IP network (Ethernet, 3G, WiFi, etc.) to communicate with the server. Communication between the devices and gateways is spread out on different frequency channels, and data rates are determined according to communication range and message duration. This selection can be managed by a LoRaWAN network infrastructure, which selects the data rate and channel for each device using an Adaptive Data Rate (ADR) scheme [26].

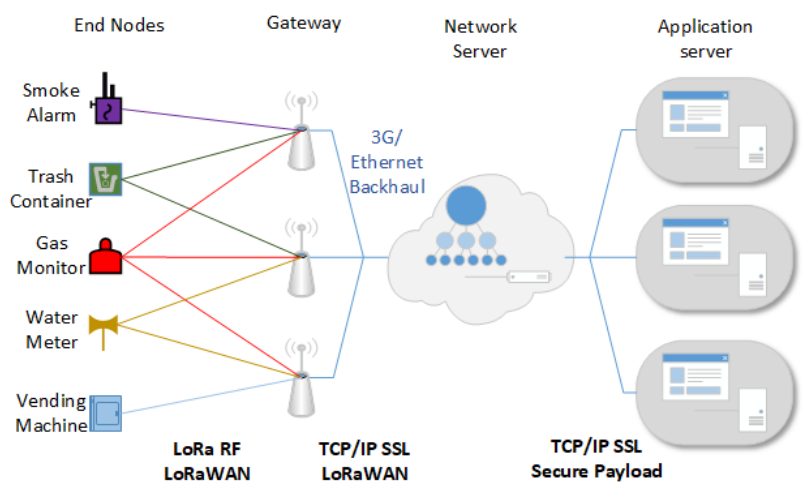

Fig. 2. LoRaWAN System Architecture

A LoRaWAN network consists of the following elements: 
- End-Device (ED): can be anything that sends or receives information. There is no real definition of an ED, but it usually refers to sensors, detectors, actuators and where sensing and controlling take place.

- Gateway (GW): is also called modem or access point. It is used to forward messages from/to the ED and NS. In LoRaWAN, EDs are not linked with the GW. Instead, any message from an ED received by the GW will be delivered to the NS.

- Network Server (NS): is the most intelligent part of the LoRaWAN network. It is responsible for:

- Monitoring the GW and ED.

- Aggregating the incoming data.

- Routing/forwarding incoming messages to the corresponding application server.

- Removing duplicates: remove duplicate messages received from one ED through multiple GW.

- In the downlink, selecting one GW based on the higher Received Signal Strength (RSS).

- Buffer downlink messages: is used to store downlink messages until the intended ED wakes up.

- Application Server (AS): It represents the application for a developer or manufacturer to parse the messages received from an ED. For example, in a cooling system application, if the temperature rises over $25^{\circ} \mathrm{c}$, it may decide to turn on the $\mathrm{A} / \mathrm{C}$ to decrease it.

\section{B. LoRaWAN Communications}

The LoRaWAN Alliance specifications define three classes for an ED, as shown in Figure 3. These classes have different capabilities to cover a wide range of applications. Each class constitutes a trade-off between battery life and network downlink communication latency. Based on the requirements, an ED can switch between classes, but class A must be implemented on all devices, by default.

1) Classes: The three classes are:

- Class A (Bi-directional EDs): it is the most energy efficient class, where an ED stays most of the time in the sleeping mode. Following every uplink phase, there are two downlink windows RX1 and RX2 to receive data with a latency of 1 second for each approximately [40].

- Class B (Bi-directional EDs with scheduled receive slots): it is the same as class A, but devices listen to incoming messages on regular intervals synchronized with a beacon.

- Class C (Bi-directional EDs with maximal receive slots): in this class, devices continuously listen for incoming messages unless transmitting (no latency). This class is used for real-time applications, where power is not constrained.

2) Connection Establishment with Security: To achieve security and integrity of the uplink and downlink messages between an ED and the GW and to preserve the NS time from reading messages contents that are relevant to another network or infrastructure, LoRaWAN defines two different keys used

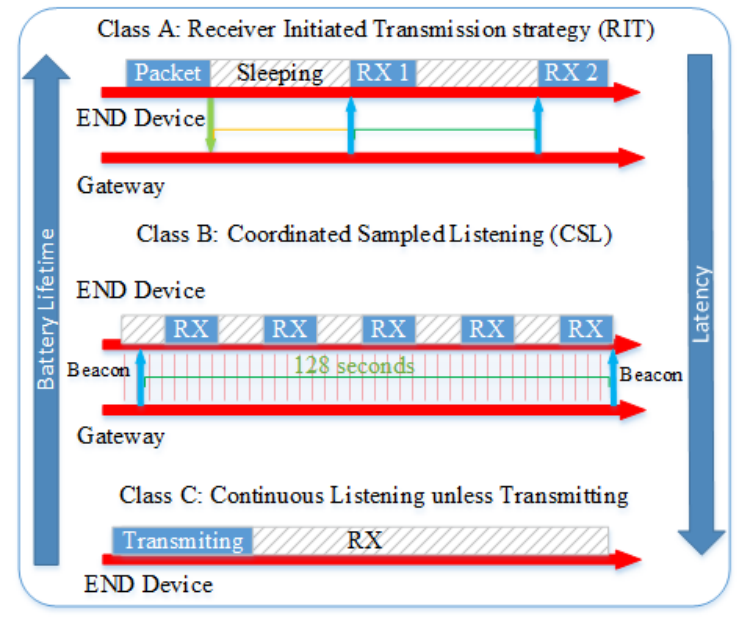

Fig. 3. LoRaWAN End-device Classes Communication

during usual message exchange as shown in Figure 4. These two keys are:

- The Network Session Key (NwkSKey): It is used to encrypt the whole frame shown in Figure 5 (headers + payload) in case a MAC-command is sent. When data are sent, this key is used to sign the message which allows the NS to verify the identity of the sender.

- The Application Session Key (AppSKey): It is used to encrypt the payload in the frame. This key does not need to be known by the NS. The AS decrypts the information using the same key.

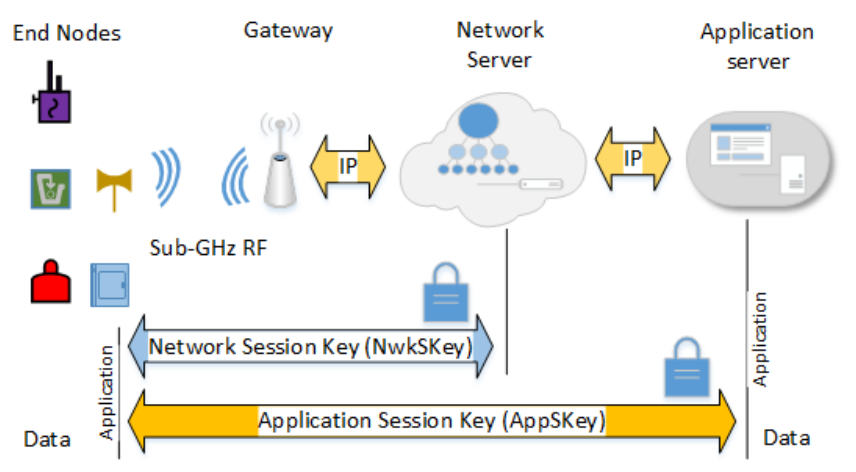

Fig. 4. Communication Exchanging and Security

3) Join the network: An ED cannot participate within the LoRaWAN network unless it has been activated. To activate the device, three types of information are required:

- Device Address (DevAddr): It consists of a 32-bit identifier which is unique within the network. This address is equivalent to an IP address on a TCP/IP network. It is present in each data frame as shown in Figure 5. This key is shared between ED, NS, and AS.

- Network Session Key (NwkSKey) mentioned above: It consists of a 128-bit AES encryption key that is unique per NS. This key is shared between an ED and the NS; it 
is used to provide message integrity and security for the communication.

- Application Session Key (AppSKey) mentioned above: It is a 128-bit AES encryption key that is unique per AS. This key is shared between an ED and the AS. It is used to encrypt and decrypt application data messages and to provide security for the application payload.

An ED can be activated to join the network using two methods. In both methods, the ED unique ID (DevEUI: is a 64-bits address equivalent to MAC address) should be known by the server before activation according to two schemes:

- Activation By Personalization (ABP): The shared keys are stored in the ED. When the ED is turned on for the first time, it can directly initiate the communication. This type of activation does not provide roaming between different network providers.

- over the Air Activation (oTAA): an ED performs a join procedure to connect to a LoRaWAN network and exchange data. In this procedure, the ED exchanges two MAC messages with the server: Join request and Join accept. During the join procedure, an ED is assigned a dynamic device address (DevAddr) and security keys are negotiated with it. This procedure is repeated every time the ED looses the connection. In this way, an ED can roam between LoRaWAN networks of different operators. This method is preferred to achieve mobility.

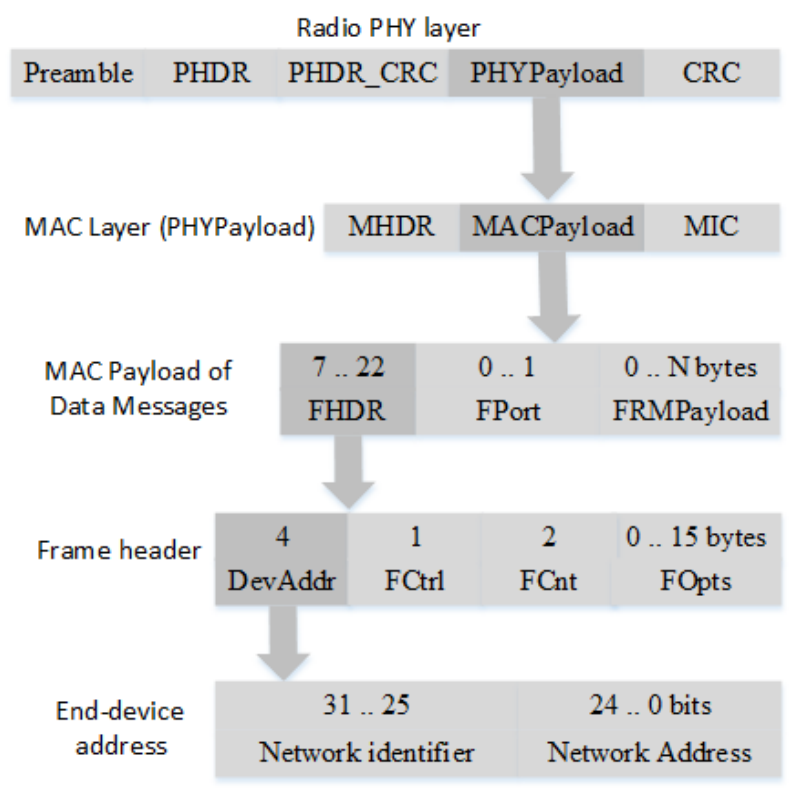

Fig. 5. LoRa Uplink PHY structure and Frame

4) Communication: After activation, an ED joins the LoRaWAN network and starts to send/receive data messages. These messages are used to transfer both MAC commands and application data, which can both be combined in a single message. LoRa allows an ED to use any possible data rate to transmit the message using an Adaptive Data Rate (ADR) scheme. This scheme is used by the network or the ED application layer to manage, adapt, and optimize the data rate of a static ED to provide the highest possible data rate. If this scheme is not enabled, the network will not control the data rate even if the received RSSI is low. In this case, the device application layer is responsible for managing the data rate. Note that this is not efficient when the radio channel attenuation changes continuously in a fast manner. An ED can benefit from the ADR scheme to increase the battery life and to maximize network capacity.

An ED and AS can request a confirmation for the message. Confirmed-data messages must be acknowledged by the receiver whereas unconfirmed-data do not require acknowledgement. In case of Figure 6 (a), the ED transmits an acknowledgement at its discretion, since the ACK is a sending operation (uplink) concerning ED. In case of Figure 6 (b), the network will send the acknowledgement using one of the receive windows opened by the ED after the sending operation. Acknowledgements are only submitted in response to the last received message and are never retransmitted.

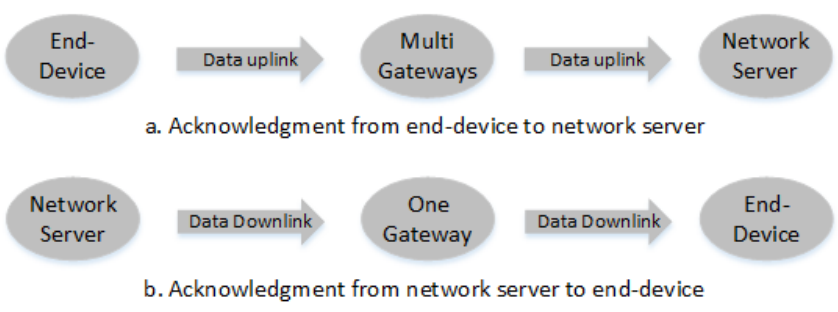

Fig. 6. Acknowledgment Message

Finally, downlink messages at physical layer are similar to uplink ones but without the CRC field, meaning that there is no payload integrity check (see Figure 7). This is to keep the message as short as possible to guarantee a minimum impact on any duty-cycle limitations of the used ISM band.

\begin{tabular}{|l|l|l|l|}
\hline Preamble & PHDR & PHDR_CRC & PHYPayload \\
\hline
\end{tabular}

Fig. 7. LoRa Downlink PHY Structure

\section{DASH7 IOT TECHNOLOGY}

The DASH7 Alliance (D7A) [41] is an open source active RFID standard for WSAN protocol. D7A complies with the ISO/IEC 18000-7 standard. ISO/IEC 18000-7 is an open standard for the license-free $433 \mathrm{MHz}$ ISM band air-interface for wireless communications. The $433 \mathrm{MHz}$ frequency provides D7A with long propagation distance and better penetration. A full OSI stack (7 OSI layers) known as D7A protocol (D7AP) is specified [29]. It provides a long range (up to $2 \mathrm{Km}$ ), and low latency with multi-year battery life to connect moving objects. Table II shows the specifications of DASH7 wireless technology.

D7A is named as BLAST network technology. The D7A features are:

- Bursty: Transmits short and sporadic sequences of data. 
TABLE II

D7A PROTOCOL SPECIFICATIONS

\begin{tabular}{|l|c|}
\hline Specification & DASH7 Technology Support \\
\hline Standard & Inherited ISO/IEC 18000-7 \\
\hline Operational Frequencies & Unlicensed ISM band \\
& $433.92,868,915 \mathrm{MHz}$ \\
\hline Modulation & 2-GFSK \\
\hline Coverage Range (Km) & $1-2$ (extend using subcontroller) \\
\hline Data Rate (kbps) & $13,55,200(16,8,4$ channels) \\
\hline Topology & Tree, Simple routing 2 hops \\
\hline
\end{tabular}

- Light: Small packet size limited to 256 bytes.

- Asynchronous: Communication is command response based, no periodic synchronization.

- Stealth: ED communicates with pre-approved GW. No need for periodic discovery beacons. This feature will be discussed in mobility section when ED moves out from the coverage of the current GW.

- Transitive: Supports mobility. ED can move seamlessly between different GWs coverages.

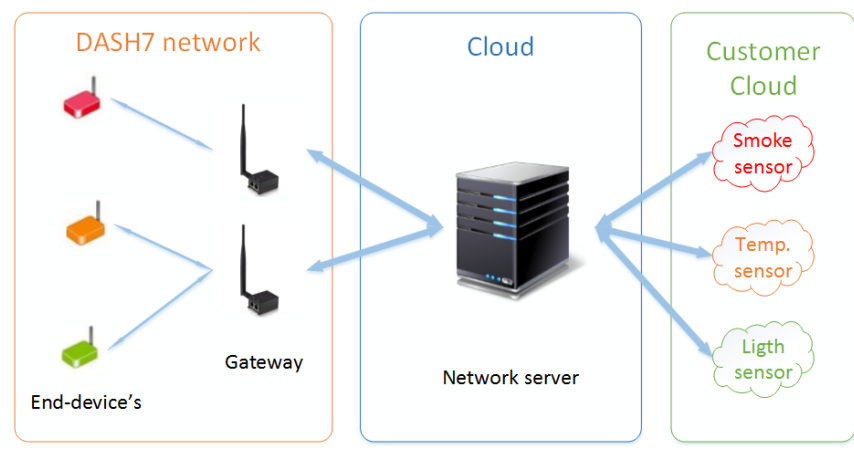

Fig. 8. DASH7 Alliance Protocol Architecture

In this part, the DASH7 Alliance protocol communication is presented along with the different layers concern with mobility to clarify the role of each one in the communication. In the uplink, EDs use CSMA/CA method which is illustrated in the physical layer. In downlink, EDs use the scan automation process that is illustrated in the data link layer. Sent/received data and ED address are presented in the Network layer section.

\section{A. Architecture}

For some basic elements, The D7AP architecture is similar to LoRa. It consists of EDs and GWs as shown in Figure 8 , and can include sub-controllers. The v1.1 specification of D7A divides the devices into three classes as shown in Table III. An ED is a simple device consisting of sensors and/or actuators with a transceiver. It gathers information and sends it to a GW when required in asynchrony mode. This device is designed to operate with minimum energy consumption (low power) and to sleep most of the time. It does not contain all D7AP features, and it uses periodic wakeup method to listen to possible incoming packets. A sub-controller device is similar to an ED and can be used as a relay for packets between an
ED and a GW. However, all D7AP features are implemented on the sub-controller device. A GW also implements all D7AP features and is always in receiving mode unless transmitting. It receives data from an ED, processes them, and forwards them to the IP-Network or transmits them to another DASH7 network. The NS shares the same features and functions as the NS in LoRaWAN, it aggregates the received data, removes duplicates when necessary, and selects the nearest GW for an ED in the downlink. Finally, the customer cloud is a program or code executed at the edge of the network. It receives the data and updates or configure the ED. Customer cloud is similar to AS in LoRaWAN.

TABLE III

D7A DEVICES CLASSES.

\begin{tabular}{|l|c|c|c|c|c|}
\hline Device Class & TX & RX & $\begin{array}{c}\text { Complete } \\
\text { Feature } \\
\text { Set }\end{array}$ & $\begin{array}{c}\text { Wake-on } \\
\text { scan } \\
\text { cycle }\end{array}$ & $\begin{array}{c}\text { Always } \\
\text { on } \\
\text { receive }\end{array}$ \\
\hline Endpoint & $\mathrm{X}$ & $\mathrm{X}$ & & $\mathrm{X}$ & \\
\hline Subcontroller & $\mathrm{X}$ & $\mathrm{X}$ & $\mathrm{X}$ & $\mathrm{X}$ & \\
\hline Gateway & $\mathrm{X}$ & $\mathrm{X}$ & $\mathrm{X}$ & & $\mathrm{X}$ \\
\hline
\end{tabular}
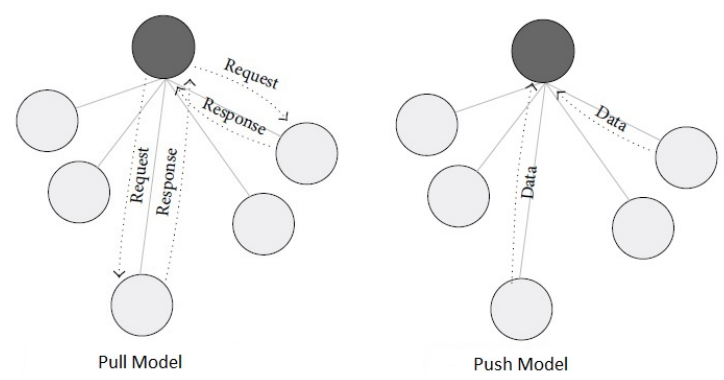

Fig. 9. DASH7 Alliance Protocol Communication Model

\section{B. D7AP Communications}

In D7AP, the communication between EDs and GWs is defined as two models shown in Figure 9. First, the pull model is a request/response mechanism. It is described by the D7A query protocol data transfer (Network layer protocol). The GW initializes it, and it is applied between the GW and the ED (More details will be given in section III-B3). The second is the push model that uses the D7AP Action Protocol (D7AActP) (Application layer protocol). D7AActP is used by an ED to send data to the GW using tag-talks-first scheme. The advantages of the push model come from the fact that it is effective in many cases to push the data. Moreover, it provides low power consumption with efficient usage of the spectrum.

D7A specifications make a correspondence between the D7A protocols and the OSI layers. D7A protocol layers are defined as follows:

1) Physical layer ( $P H Y)$ : This layer encompasses the modulation, spectrum and channel coding characteristics [29]. All data traffic in D7A has the frame structure shown in Figure 10. The packet incorporates the power ramp-up and ramp-down 
that are used to meet the bandstop channel requirements. The preamble consists of a series of binary symbols (32-bit for base \& normal or 48-bit for high-rate \& blink channels) that are used to calibrate data rate circuits on the receiver. Sync Word is a 16 binary symbols block used to align the packet payload that contains the data defined by the upper layers. The

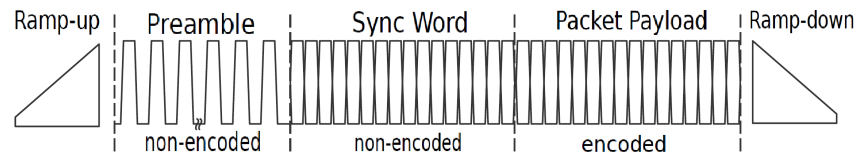

Fig. 10. D7A General Frame Structure [29]

protocol defines different channel classes: low-rate, normal and high-rate as shown in Table IV.

TABLE IV

D7AP Modulation Schemes USING 2-(G)FSK [42]

\begin{tabular}{|c|c|c|c|c|}
\hline $\begin{array}{c}\text { Channel } \\
\text { Class }\end{array}$ & $\begin{array}{c}\text { Channel } \\
\text { Spacing } \\
\text { (MHz) }\end{array}$ & $\begin{array}{c}\text { Symbol } \\
\text { Rate } \\
\text { (kbps) }\end{array}$ & $\begin{array}{c}\text { Modulation } \\
\text { Index }\end{array}$ & $\begin{array}{c}\text { Frequency } \\
\text { Deviation } \\
\text { (KHz) }\end{array}$ \\
\hline Lo-Rate & 0.025 & 9.6 & 1 & \pm 4.8 \\
\hline Normal & 0.200 & 55.55 & 1.8 & \pm 50 \\
\hline Hi-Rate & 0.200 & 166.67 & 0.5 & \pm 41.667 \\
\hline
\end{tabular}

EDs use CSMA/CA method to access the channel and transmit data. Before starting the transmission process an ED guards (reserves) the channel, as shown in Figure 11, for the period of transmission. This period is extended if the transmission time is greater than or equal to the guard interval $\left(T_{G}\right)$. The channel guard is extended by $T_{G}$ after the transmission, but if the transmission time is less than $T_{G}$, there is no extension. In addition, let $T_{T}$ be the minimal duration of the silent period between two transmissions, and $T_{S}$ be the silent period, we have $T_{T}<T_{S}<T_{G}$. The values of the channel guarding constants are given in Table $\mathrm{V}$.

TABLE V

Channel Guarding Constants

\begin{tabular}{|c|c|c|}
\hline $\begin{array}{c}\text { Constant } \\
\text { Parameter }\end{array}$ & \multicolumn{1}{|c|}{ Description } & $\begin{array}{c}\text { Value } \\
\mathbf{T i}=(\sim \mathbf{0 . 9 7 7} \mathbf{~ m s})\end{array}$ \\
\hline$T_{G}$ & Channel Guarding Interval & $5 \mathrm{Ti}$ \\
\hline$T_{T}$ & Channel Turnaround Interval & $2 \mathrm{Ti}$ \\
\hline
\end{tabular}

2) Data Link Layer (DLL): This layer specifies the data link addressing; The fixed unique ID (UID) is a 64-bit value and must be unique to every D7A device. The dynamic networkunique virtual ID (VID) is a 16-bit ID supplied by the network administrator which is unique within the network. DLL defines the transmission, reception, scan automation and multiple access processes. Two types of frames shown in Figure 12 are held in this layer:

- Background frame: a fixed length 6-byte frame, preceded by a sync word of class 0 .

- Foreground frame: a variable length, up to 255 bytes, preceded by a sync word of class 1 .
A subnet parameter consisting of 4-bit specifier and a 4bit mask is used to filter the incoming frames. Each device contains an internal subnet value known as the device subnet which is compared with the value of the received frame subnet known as frame subnet. A Cyclic Redundancy Check (CRC) is used to check the integrity of the frame. The Target Address (TADR) parameter holds the address of the destination. The type of address used (UID or VID) on TADR is specified in the first 2 bits of the CTRL parameter, which also holds the estimated radiated power of the transmitter in the following 6 bits.

DLL defines the first level frame filtration, where three steps filter incoming frames are:

- Cyclic Redundancy Check (CRC16).

- Subnet matching and link quality.

- Device ID matches.

\section{Device to Device Communication}

The D7A protocol also supports device to device communication, which is defined in this layer using the concept of Access Profile (AP) and Access Class (AC). AP defines the subnet, transmission time-out, period of automatic scanning, and the number of the sub-bands to scan or transmit on. Table VI shows the parameters that allow accessing a remote device through a channel scanned by the latter device. It is composed of 4 sub-profiles and a list of 8 sub-bands. All the sub-bands share the same channel header and allow the node to communicate on a group of channels. A sub-profile is a combination of the sub-bands and a scheduling time as shown in Table VII. This combination is described in the one-byte bitmap. The AC is divided into two fields: the Access Specifier (AS) and the Access Mask (AM), as shown in Table VIII. AS is the index of the D7A file. This file contains the AP. The APs are not exchanged between devices, and only the ACs are transferred. Before deploying the network, the network administrator must set up the AP, sub-profiles and has to link an $\mathrm{AC}$ to each profile. This configuration must be known by all devices in the network and be unique.

To communicate with Device $B$, Device $A$ must use the AC of Device $B$. The application layer of $A$ provides the address of $B$ to initiate a dialog using one of the channels that $B$ scans [29] (refer to specification v1.1 section 7.3 and subsection 8.4.5). Device $B$, using its own $\mathrm{AC}$, will scan the associate channel list during the automated scan routine every $T_{S C H E D} \mathrm{~ms}$. If $B$ detects the message, a dialogue will be opened between them to exchange requests and responses $(B$ extracts the address of $A$ from the "Origin" field in D7ANP network layer) and it will be closed when they finish. In case $B$ changes its class, $A$ is no more able to communicate unless $B$ informs $A$ about the changes or $B$ initiates a dialog with $A$. In the D7A specification, there is no indication of how this notification is performed. But all packets sent by a device contain the $\mathrm{AC}$, so if $A$ receives a packet from $B$, it will get the new class of this device. 

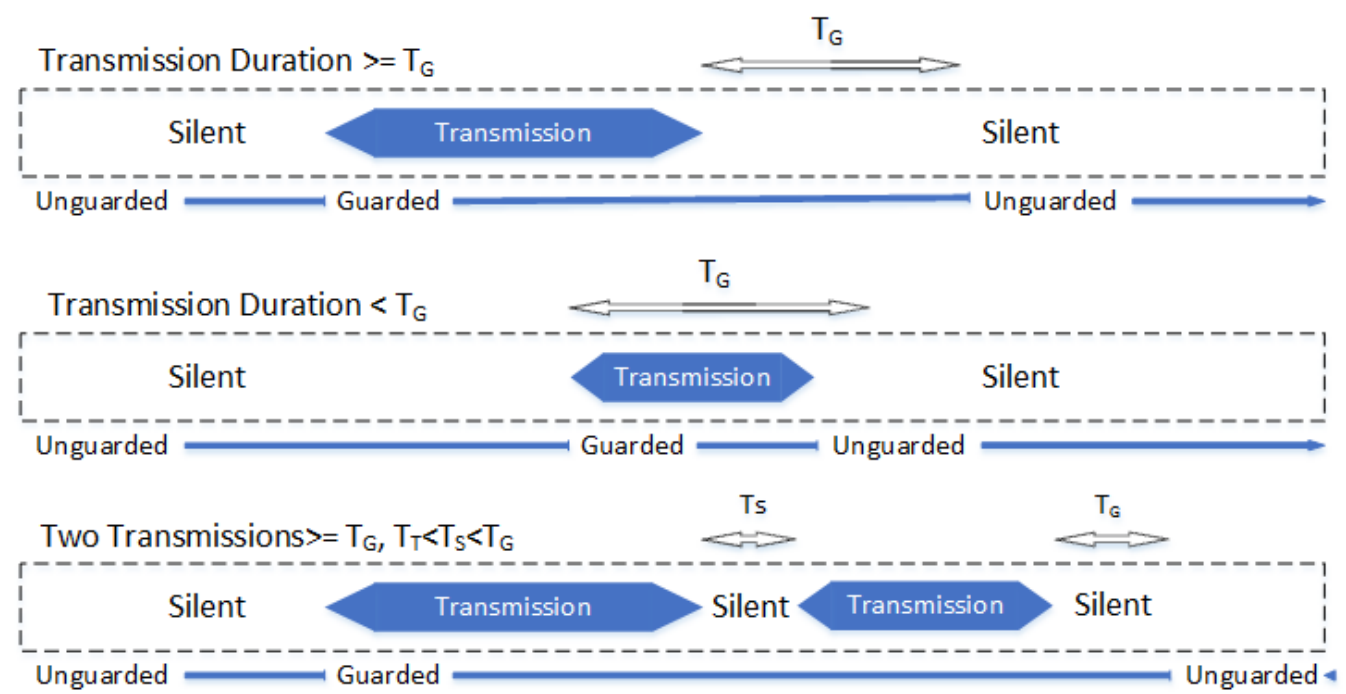

Fig. 11. Channel Guarding

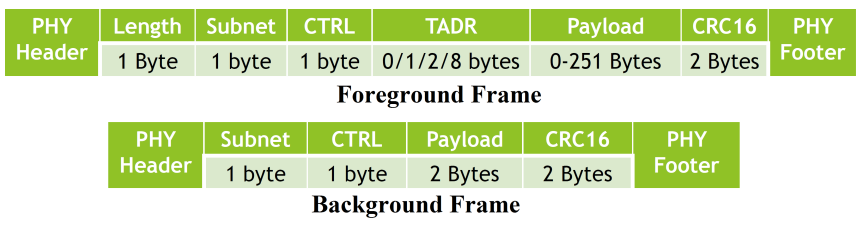

Fig. 12. Foreground and Background Frame Structure

TABLE VI

ACCESS PROFILE

\begin{tabular}{|c|c|c|}
\hline Parameter & $\begin{array}{c}\text { Size } \\
\text { (byte) }\end{array}$ & Description \\
\hline CH & 1 & Channel Header \\
\hline SP & $4 \times 2$ & Sub-profiles 0 to 3 \\
\hline SB & $8 \times 7$ & Sub-bands 0 to 7 \\
\hline
\end{tabular}

\section{Scan Automation Process}

EDs use scan automation process to receive data messages. Scan automation defines scan timeout, foreground scan, background scan, reset and restart.

Scan Timeout: $T_{o}$ specifies the duration of the period during which a device tries to receive a DLL frame. If the value of $T_{o}$ is not defined by a DLL scan automation process or upper layer, its value will be dependent on the channel class, the timing tolerance of the device, and the maximum length of the PHY packet preamble.

Foreground Scan : If $T_{S C H E D}=0$, the device will continuously scan the channel list, in parallel. This scan is only paused when the upper layer preempts the DLL frame to transmit or receive. In this case, scan timeout $\left(T_{o}\right)$ will be set to 0 .

Background Scan : If $T_{S C H E D}>0$, an independent schedule is set to generate a regular scan, it starts events at the $T_{S C H E D}$ rate. When the device cannot run the scan automation, these scans events will be masked. A background scan of the scan automation channel list is started on every unmasked scan
TABLE VII

ACCESS SUb-PRofILE

\begin{tabular}{|c|c|c|}
\hline Sub-band Bitmap & 1 byte & Bitmap of used sub-bands \\
\hline$T_{S C H E D}$ & 1 byte & $\begin{array}{c}\text { Scan automation period } \\
\text { (compressed format) }\end{array}$ \\
\hline
\end{tabular}

TABLE VIII

ACCESS CLASS

\begin{tabular}{|l|l|l|l|l|l|l|l|}
\hline b7 & b6 & b5 & b4 & b3 & b2 & b1 & b0 \\
\hline \multicolumn{2}{|l|}{ Access Specifier } & \multicolumn{4}{|l}{ Access Mask } \\
\hline
\end{tabular}

event. Upper layers select the value of $T_{0}$.

Reset and Restart: The scan resets

- when the scan automation channel list is consumed.

- stopped by the upper layer to transmit or receive.

After the scan resets, it is restarted

- immediately if $T_{S C H E D}=0$

- at the next scheduler event if $T_{S C H E D}>0$

3) Network Layer (NWL): This layer defines the background network protocol, and the foreground network protocol.

The D7A Advertisement Protocol (D7AAdvP) is a transmission-only background network protocol (pull communicational model). It is used for rapid and ad-hoc group synchronization. D7AAdvP is defined in D7AP as a low-power wakeup mechanism used by a gateway or a sub-controller to query EDs. The GW or sub-controller starts by continuously transmitting the D7AAdvP to flood the channel with background advertising frames for a duration that depends on the EDs AP [29]. Each frame contains the Estimated Time of Arrival (ETA), which is the time to send the foreground frame that includes the Application Layer Protocol (ALP) command. This value is decremented in the subsequent background advertising frames until reaching zero. EDs are 
configured to schedule a background scan (frame of class 0 ) at a specific rate. At a particular time that corresponds to each ED configuration, an ED wakes up and starts listening by scanning the channel for incoming background frame. When the frame is received, the ED extracts the ETA value and returns to sleeping mode until the time (ETA) is elapsed. Then, the ED wakes up and scans for foreground frames (frame of class 1) to receive and respond the request. This mechanism leads to very low power consumption for ad-hoc synchronization. Synchronization train is shown in Figure 13.

The foreground network protocols are used for responses, queries and beacons:

- D7A Network Protocol (D7ANP) is an addressable (unicast, broadcast, multicast and any-cast), and routable protocol. It is used by D7A Query Protocol in the transport layer. It supports two-hop routing and security in the network layer.

- D7A DataStream Protocol (D7ADP) which is used by the ALP, is a generic data encapsulation protocol. This protocol does not contain information about routing or addressing.

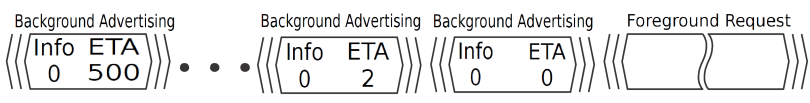

Fig. 13. Synchronization Train Chain [29]

4) Upper Layers: The Transport Layer (TPL) provides end-to-end communication services. It defines the concept of request-response, and a method for acknowledging single and group requests.

The Session Layer (SEL) indicates which events may trigger session initiation or scheduling. It defines the concept of QoS and the method for queuing, transmitting, re-transmitting, scheduling, and receiving upper layer requests.

The ALP contains the application API. The latter defines a standard method to manage Data Elements by the application.

\section{NB-IOT TECHNOLOGY}

Narrow Band Internet of Things (NB-IoT) is a part of release 13 [36]. It was setup by 3GPP in Cellular systems in support for ultra-low complexity and low throughput Internet of Things (CIoT). It defines a new radio access technology that can be integrated into the LTE standard. NB-IoT is built from existing LTE functions, but many features have been removed to keep this standard as simple as possible to reduce device cost and minimize battery consumption. This optimization includes removing handover, carrier aggregation, measurements to monitor the channel quality, and dual connectivity. NB-IoT operates on the same licensed frequencies used by LTE and employs QPSK and BPSK modulations. Table IX shows the specifications of NB-IoT.

Physical layer is designed to fit in $200 \mathrm{kHz}$ system bandwidth used by both uplink and downlink. This enables NB-IoT to gain the feature of deployment in the GSM carrier as standalone, and in LTE as in-band or guard-band as shown in the Figure 14.

- Standalone: Replacing a GSM carrier with an NB-IoT cell.

- Guard-Band: Benefit from unused resource blocks within the guard-band of LTE carrier.

- In-Band: Use one or more Physical Resource Blocks (PRBs) that are reserved for NB-IoT.

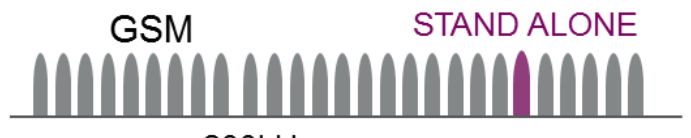

$200 \mathrm{kHz}$
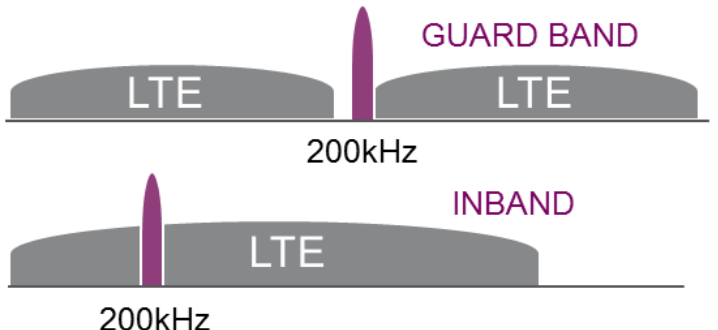

Fig. 14. NB-IoT Deployment Modes [43]

TABLE IX

NB-IOT PROTOCOL SPECIFICATIONS

\begin{tabular}{|l|c|}
\hline Specification & NB-IoT Technology Support \\
\hline Standard & 3gpp (release 2015) \\
\hline Operational Frequencies & Same LTE band \\
\hline Modulation & QPSK \& BPSK \\
\hline Coverage Range $(\mathbf{K m})$ & $<15$ \\
\hline Data Rate $(\mathbf{k b p s )}$ & $\sim 50$ \\
\hline Topology & Star \\
\hline
\end{tabular}

\section{A. Architecture}

NB-IoT uses the same network architecture as in LTE network but with some optimizations to meet the requirements of IoT massive users. NB-IoT architecture is based on the Evolved Packet System (EPS) as shown in Figure 15. A new node has been added to the architecture, known as Service Capability Exposure Function (SCEF), which is designed for machine type data. Two optimizations are defined for CIoT in EPS: Control plane CIoT EPS optimization (red lines), and user plane CIoT EPS optimization (blue line). Both optimizations may be used for sending data to the correspondent application. On the user plane, the blue line, IP and non-IP data are transferred in the same way as for the conventional data traffic, i.e., over radio bearers via the Serving Gateway (SGW) and the Packet Data Network Gateway (PGW) to reach the application server. With the control plane, the red lines, the radio communications between the user equipment (End-Device) and MME are handled by the evolved UMTS terrestrial radio access network (E-UTRAN), which consists 
TABLE X

Channels and Signals [45]

\begin{tabular}{|c|c|c|}
\hline \multicolumn{2}{|c|}{ Channel } & Usage \\
\hline \multirow{4}{*}{ UL } & $\begin{array}{c}\text { Narrowband Physical Uplink } \\
\text { Shared Channel (NPUSCH) }\end{array}$ & Uplink dedicated data \\
\cline { 2 - 3 } & $\begin{array}{c}\text { Narrowband Physical Random } \\
\text { Access Channel (NPRACH) }\end{array}$ & Random access \\
\hline \multirow{4}{*}{ DL } & $\begin{array}{c}\text { Narrowband Physical Downlink } \\
\text { Control Channel (NPDCCH) }\end{array}$ & $\begin{array}{c}\text { Uplink and downlink } \\
\text { scheduling information }\end{array}$ \\
\cline { 2 - 3 } & $\begin{array}{c}\text { Narrowband Physical Downlink } \\
\text { Shared Channel (NPDSCH) }\end{array}$ & $\begin{array}{c}\text { Downlink dedicated and } \\
\text { common data }\end{array}$ \\
\cline { 2 - 3 } & $\begin{array}{c}\text { Narrowband Physical } \\
\text { Broadcast Channel (NPBCH) }\end{array}$ & $\begin{array}{c}\text { Master information for } \\
\text { system access }\end{array}$ \\
\cline { 2 - 3 } & Narrowband Synchronization & Time and frequency \\
& Signal (NPSS/NSSS) & synchronization \\
\hline
\end{tabular}

of the evolved based stations known as eNodeB or eNB (Gateway). Then, uplink data are transmitted to the SGW that forwards them to the PGW. Non-IP data will be sent using SCEF, which is the new node responsible for delivering nonIP data over control plane and providing an interface for the network services (authentication and authorization, discovery and access network capabilities).

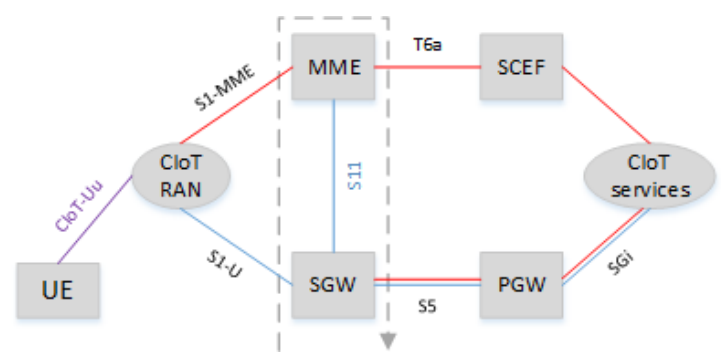

Fig. 15. NB-IoT Architecture

There is no difference in the access network architecture compared to LTE [44]. The GW is connected to the MME and S-GW using the S1 interface as shown in Figure 16. GWs are connected together with the X2 interface although there is no handover, this interface enables a fast connection resuming when ED change from IDLE_STATE to RCC_CONNECTION. This will be explained in the mobility section below.

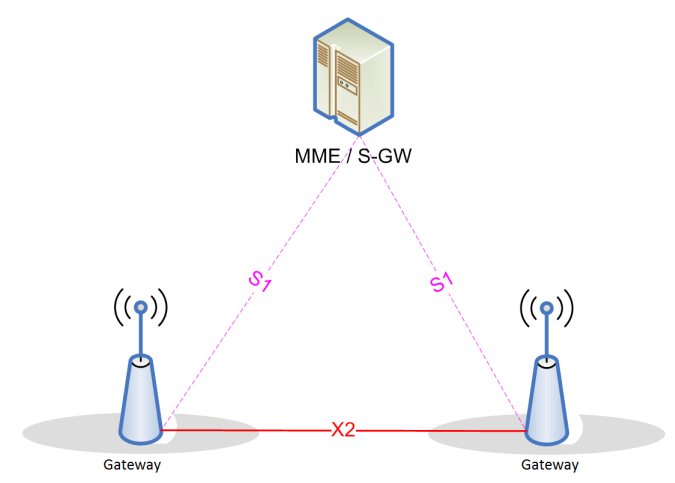

Fig. 16. Network Architecture Towards the Air Interface [44]

\section{B. NB-IoT Communications}

In this subsection, we focus on the physical layer and resource mapping. A summary will be given to different channels and signals for downlink (DL) and uplink (UL) that are used by NB-IoT as shown in Table X.

1) NPSS/NSSS: In case of in-band and guard-band mode, NPSS/NSSS signals can be only transmitted in the certain subset of the available LTE PRB locations, as shown in Figure 17. This is due to the frequency offset between the DC carrier and the centre of the NB-IoT carrier that should be held within $\pm 7 \mathrm{kHz}$ range, to ensure efficient cell searching. NPSS is transmitted every $10 \mathrm{~ms}$ and NSSS every $20 \mathrm{~ms}$.

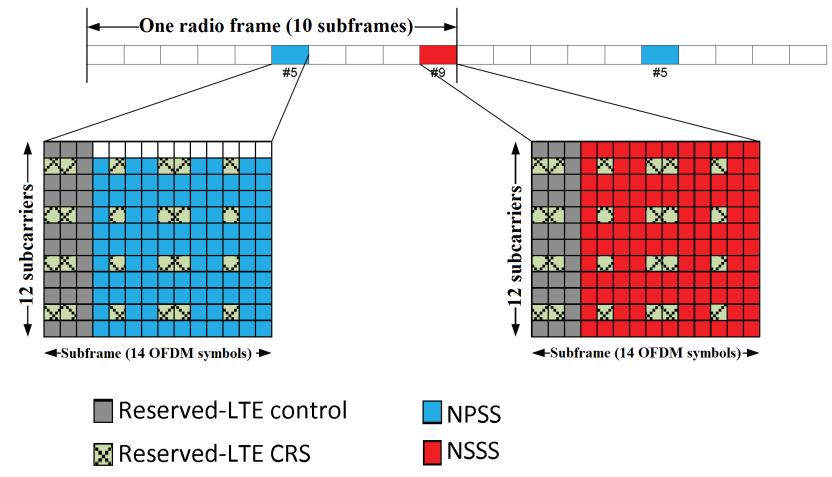

Fig. 17. Primary and Secondary Synchronization Signals Transmission [45]

2) NPBCH: It is responsible for transmitting the NarrowBand Master Information Block (MIB-NB) over an $80 \mathrm{~ms}$ block. This transmission is repeated 8 times, where MIBNB is transmitted precisely without any content change for $640 \mathrm{~ms}$ using QPSK modulation, to ensure that the block is received in extreme coverage conditions. Figure 18 shows the $\mathrm{NPBCH}$ transmission and the location of the NRS signals. MIB-NB is a 50-bits size block that contains 16-bit CRC and spare bits. This block is used to provide an NB-IoT ED with the main information like System Frame Number (SFN). Also, it provides the operational mode, channel raster, LTE Cell-specific Reference Signal (CRS), and System Information Block (SIB) scheduling.

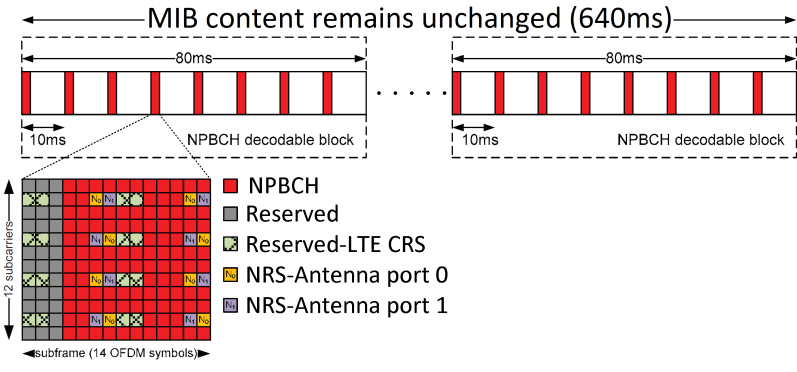

Fig. 18. NPBCH Transmission [45]

3) NPDCCH: This channel is used to carry downlink control information, like paging or system information. Depending on the used NPDCCH format as shown in Table XI, data 
may be carried by one or an aggregation of two subsequent Narrow Band Control Channel Elements (NCCEs) during a sub-frame. Each NCCF consists of six sub-carriers in a subframe as shown in Figure 19. The search space defines which NPDCCH transmission sub-frame an ED is searching for. Repetition of transmissions is used in NB-IoT to achieve coverage enhancement. Depending on the coverage level, each ED is configured to transmit NPDCCH several times based on the $\mathrm{R}_{\max }$ which is chosen from up to 2048 (value $2^{n}$ ). The number of repeated transmissions is also indicated in the DCI as illustrated in Table XII. Then, an ED can determine the end of the NPDCCH transmission when it successfully decodes the NPDCCH before the last repetition [45].

TABLE XI

NUMBER OF AGGREGATED NCCES FOR EACH NPDCCH FORMAT

\begin{tabular}{|c|c|}
\hline NPDCCH Format & Number of NCCEs \\
\hline 0 & 1 \\
\hline 1 & 2 \\
\hline
\end{tabular}

TABLE XII

DCI FORMATS

\begin{tabular}{|c|c|}
\hline \multicolumn{2}{|c|}{ DCI Formats } \\
\hline N0 & NPUSCH Scheduling \\
\hline N1 & $\begin{array}{c}\text { NPDSCH Scheduling } \\
\text { and NPDCCH Order }\end{array}$ \\
\hline N2 & Paging and direct indication \\
\hline
\end{tabular}
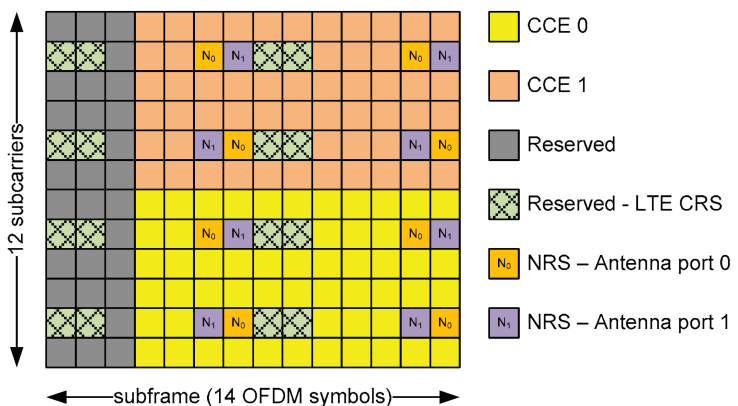

Fig. 19. CCE Allocation in NPDCCH (in-band operation mode) [45]

4) NPDSCH: This channel is scheduled after NPDCH, to give time to end-devices to decode NPDCCH. This delay, which is at least $4 \mathrm{~ms}$, starts from the end of the NPDCCH to the beginning of NPDSCH and reduces the complexity of NBIoT end-devices. NPDSCH employs the whole 12 sub-carriers in the downlink bandwidth. Only single HARQ process, which is adaptive and asynchronous, is supported in the downlink.

5) NPRACH: This signalling channel can be used by ED in the random access channel procedure for cell accessing, where the preamble is transmitted.

A preamble is based on a single sub-carrier of a single group, with frequency hopping for a single user as shown in Figure 20. Each symbol group has a Cyclic Prefix (CP) followed by five symbols. Hopping is between groups of
TABLE XIII

NPDSH SPECIFICATIONS

\begin{tabular}{|c|c|c|}
\hline Modulation & \multicolumn{2}{|c|}{ only QPSK } \\
\hline $\begin{array}{c}\text { Maximum Transport } \\
\text { Block size (TBS) }\end{array}$ & \multicolumn{2}{|c|}{680 bits } \\
\hline Channel coding & \multicolumn{2}{|c|}{ TBCC } \\
\hline Redundancy & \multicolumn{2}{|c|}{ not supported } \\
\hline Error detection & \multicolumn{2}{|c|}{ Supported using 24-bit CRC } \\
\hline \multirow{2}{*}{ Download Schemes } & \multirow{2}{*}{\multicolumn{2}{|c|}{$\begin{array}{c}\text { Using one antenna port (port 0) } \\
\text { Using Space-Frequence block } \\
\text { coding (SFBC) for two } \\
\text { antenna port (port } 0 \text { and } 1 \text { ) }\end{array}$}} \\
\hline & & \\
\hline \multirow{2}{*}{ Data Rate (kbps) } & Instantaneous Peak & 170 \\
\hline & Sustained Peak & 26.2 \\
\hline Rmax & $\begin{array}{r}1,2,4,8,16,32,6 \\
384,512,768,10\end{array}$ & $\begin{array}{l}128,192,256, \\
4,1536,2048\end{array}$ \\
\hline
\end{tabular}

symbols whereas pseudo-random hopping concerns repetitions of groups. Different cell sizes can be achieved when using subcarrier spacing of $3.75 \mathrm{kHz}$, with a symbol length of $267 \mu \mathrm{s}$, and two cyclic prefix lengths: $66.7 \mu \mathrm{s}(10 \mathrm{Km})$ and $267 \mu \mathrm{s}$ (35 Km). NPRACH has three resource configurations within a cell, each of which corresponds to a different coverage level. A resource configuration is given by:

- Periodicity.

- Number of repetitions, Up to 2048 and 128 times in DL and UL to enhance coverage.

- Starting time.

- Frequency location.

- Number of sub-carriers, it can be $12,24,36,48$.

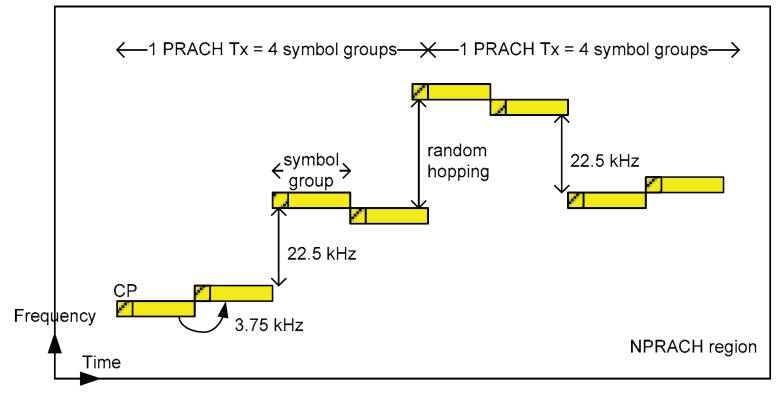

Fig. 20. NPRACH channel [45]

6) NPUSCH: This channel is designed to carry uplink data and send HARQ Ack/Nack. It provides extended coverage, long battery life, and massive capacity. This channel has two formats: Format 1 is used to send uplink data (maximum transport block: 1000 bits). Table XIV shows the smallest amount of time-frequency resource units (RU). For RUs with one subcarrier, BPSK and QPSK may be used, while for all other RUs, QPSK is applied. Format 2 is used in signalling HARQ acknowledgement for the downlink channel NPDSCH. In this case, the modulation scheme is always BPSK. It always uses one sub-carrier with a length of 4 slots. In the case of a $3.75 \mathrm{kHz}$ spacing, an RU has an $8 \mathrm{~ms}$ duration whereas in 15 $\mathrm{kHz}$ sub-carrier the duration is $2 \mathrm{~ms}$. NPUSCH supports the following features: 
- Large transport block.

- Time-domain repetition. It helps in extending coverage and channel estimation as explained before.

- Single-tone transmission $(3.75 \mathrm{kHz}$ or $15 \mathrm{kHz}$ sub-carrier space), and multi-tone transmissions (15 kHz sub-carrier spacing).

- low peak-to-average-power ratio (PAPR) modulation schemes ( $\pi / 2$-BPSK and $\pi / 2$-QPSK) for single-tone transmission.

TABLE XIV

NPUSCH RU DEFINITION

\begin{tabular}{|c|c|c|c|}
\hline $\begin{array}{c}\text { Subcarrier } \\
\text { spacing } \\
(\mathrm{kHz})\end{array}$ & $\begin{array}{c}\text { Number } \\
\text { of } \\
\text { Tones }\end{array}$ & $\begin{array}{c}\text { Number of } \\
\text { SC-FDMA } \\
\text { symbols }\end{array}$ & $\begin{array}{c}\text { Transmission } \\
\text { time interval } \\
(\mathrm{ms})\end{array}$ \\
\hline \multirow{3}{*}{15} & 1 & 112 & 8 \\
\cline { 2 - 4 } & 3 & 56 & 4 \\
\cline { 2 - 4 } & 6 & 28 & 2 \\
\hline 3.75 & 12 & 14 & 1 \\
\hline
\end{tabular}

Protocol Stack: NB-IoT protocol stack starts with the protocol layers used in LTE protocols. These layers have been reduced and optimized to meet the requirements of NB-IoT. This protocol is built on a well-established fundamental and can be viewed as a new air interface technology. NB-IoT protocol stacks shown in Figure 21 look the same as for LTE but with optimized functionalities.

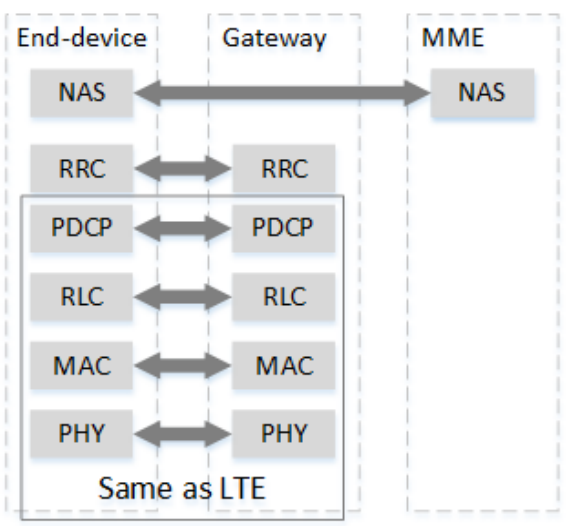

Fig. 21. NB-IoT Protocol Stack

System Information: These blocks are used to broadcast information for all EDs within the range of the GW. Table XV illustrates a set of SIBs used in NB-IoT and defines them. In case of system information acquisition or changes, ED is returned to the IDLE state if connected. Even if NB-IoT is deployed in-band with LTE, EDs will ignore SIBs from LTE.

\section{Mobility management}

After presenting the specifications of LoRaWAN, DASH7 Alliance (D7A), and NB-IoT, we can note that the mobility has not been highlighted in those IoT environments. The requirement of mobility in such environments is different from the mobility management found in IETF [46] protocols.
TABLE XV

SYSTEM INFORMATION BLOCKS CONTENT

\begin{tabular}{|c|c|}
\hline System Information Block & Content \\
\hline MIB-NB & $\begin{array}{c}\text { Essential information required to } \\
\text { receive further system information }\end{array}$ \\
\hline SIBType1-NB & $\begin{array}{c}\text { Cell access and selection, } \\
\text { other SIB scheduling }\end{array}$ \\
\hline SIBType2-NB & $\begin{array}{c}\text { Radio resource configuration } \\
\text { information }\end{array}$ \\
\hline SIBType3-NB & $\begin{array}{c}\text { Cell re-selection information for } \\
\text { intra-frequency, inter-frequency }\end{array}$ \\
\hline SIBType4-NB & $\begin{array}{c}\text { Neighboring cell related } \\
\text { information relevant for } \\
\text { intra-frequency cell re-selection }\end{array}$ \\
\hline SIBType5-NB & $\begin{array}{c}\text { Neighboring cell related } \\
\text { information relevant for } \\
\text { inter-frequency cell re-selection } \\
\text { Access Barring parameters }\end{array}$ \\
\hline SIBType14-NB & $\begin{array}{c}\text { Information related to GPS time and } \\
\text { Coordinated Universal Time (UTC) }\end{array}$ \\
\hline SIBType16-NB
\end{tabular}

Mobility management in IP networks consists in providing seamless connectivity during IP handover (either soft or hard handover), whereas the mobility in IoT refers to ensuring the delivery of information on demand and during movement. In the following, we show the mechanism that ED of each technology follows to ensure data delivered while moving. In this section, we consider the mobility of an ED within those three technologies, i.e. when an ED is moving in an IoT environment. As stated above, beyond ubiquity of EDs in IoT environments, there is an increase in IoT applications that require mobility. ED may consist of a human carrying a smart phone, autonomous vehicle, robot, drone, etc. Thus, investigating and supporting mobility is a significant requirement for a wide range of IoT applications. Here, we mainly focus on the mobility of an ED moving within one technology, under different GWs that belonging to a single network operator as shown in Fig. 22. In the following, we will explain the switching procedure achieved by ED after losing connection with current GW, in order to connect with a new GW.

In the following, we denote by "Uplink" the data frame transmitted from an ED to the GW, and by "Downlink" the data frame that is transmitted from the GW to an ED. A typical scenario considered in this case is the handover of an ED from a gateway to another one in two cases: first, a message is transmitted in uplink and second, a message is transmitted in downlink to the ED. In the following, we investigate in details this scenario for the three IoT technologies: LoRaWAN, D7A, and NB-IoT.

\section{A. LoRaWAN}

In LoRaWAN, a GW is seamless to an $\mathrm{ED}$, where an ED sends data when available without considering location change, movement, and speed of motion. On the second side, any GW that receives the message will forward it to the NS. In this protocol, to clarify mobility, we take into consideration the class of the node and the transmitting/receiving (uplink/downlink) process. 


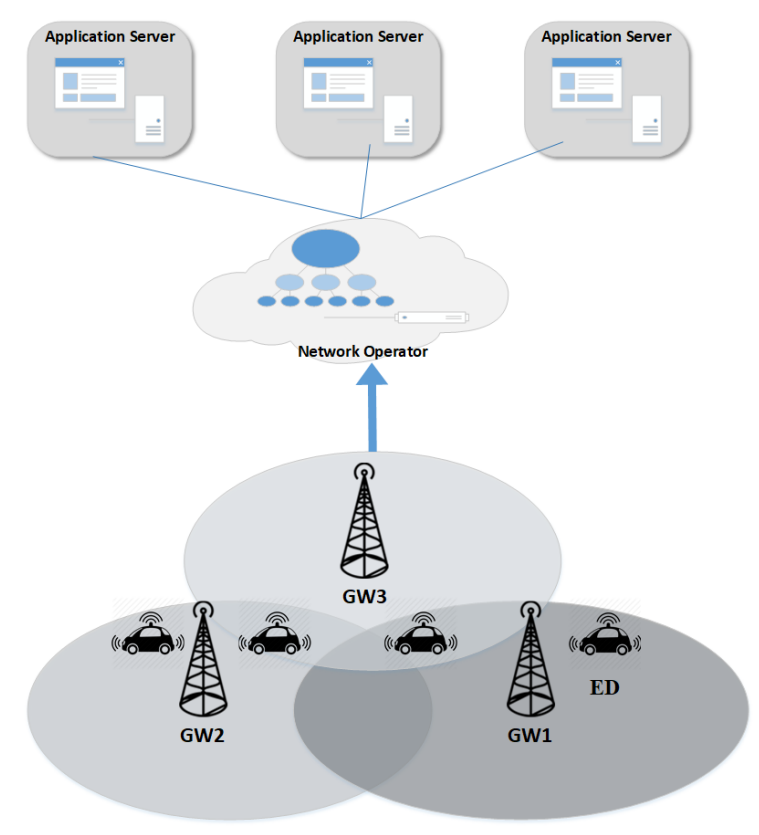

Fig. 22. Mobility scenario within IoT environment

1) Class A: An ED sends an Uplink message to an NS relayed by one or more GWs. Uplink messages can be sent at any time using the Aloha method. Any GW in the range of the ED that receives the message will forward it to the NS. The GW attaches the RSSI to every received message from any ED before delivering it to the NS. The RSSI value is used to indicate to the NS the nearest GW from the ED. A forward table is created on the NS that contains the ED and GW addresses. This table will be used later by the NS to forward a downlink message to an ED. LoRaWAN specifications (release 2016) do not describe the transmission of multicast messages from NS to more than one ED [26]. The message format at the physical layer is shown in Figure 5, where the radio transceiver inserts the LoRa Physical Header (PHDR), CRC header (PHDR_CRC), and payload CRC field (used to protect payload integrity).

Two receive windows are opened by an ED after every uplink transmission to receive the downlink message. The NS sends the downlink message to the ED relayed by only one GW selected from the forward table. Data that are intended for a particular ED are queued in the NS until receiving a message from this device. This indicates that the device has wake-up and that two receiving windows have been opened. From the uplink message, an NS can determine the location of the device as explained previously. Then, the NS initiates the transmission just following one second after receiving uplink message from the ED. During one of the receive windows, if a preamble is detected, the radio receiver stays active until the downlink frame is demodulated. If an ED detects and demodulates the downlink message during the first receiving window, this ED will check the address and Message Integrity Code (MIC). If this message belongs to this ED, RX2 will not be opened, and the ED enters the sleep mode.
2) CLass B: It extends class $\mathrm{A}$ by adding synchronized reception windows. An ED is synchronized using the timesynchronization beacons transmitted by the GW. Class B is intended for mobile and fixed devices. The primary purpose is to have a synchronized device that listens on fixed time intervals to receive the messages. The decision of switching from class A to B is triggered from the application layer. If the network controls the decision, the application layer on the ED must be able to recognize the request of the application server for switching. When ED changes its place, it is configured to send an uplink message to the NS (even an empty message) to update its new location in the forwarding table of the NS. While location of ED changes to a GW that does not support class $\mathrm{B}$, the ED directly switches to class A.

3) CLass C: An ED always listens on RX2 window slot unless it transmits or receives on RX1. Class $\mathrm{C}$ device implements the same receiving windows (RX1, RX2) as class $\mathrm{A}$, but the RX2 window is not closed unless transmitting as shown in Figure 23. A short RX2 window is also opened directly after the end of transmission and before the beginning of the RX1 receiving window, using the same frequency and data rate of RX2 . Any time NS sends a downlink message, a class $\mathrm{C}$ device can receive. When location changes, ED sends an uplink message to inform the NS about the changes.

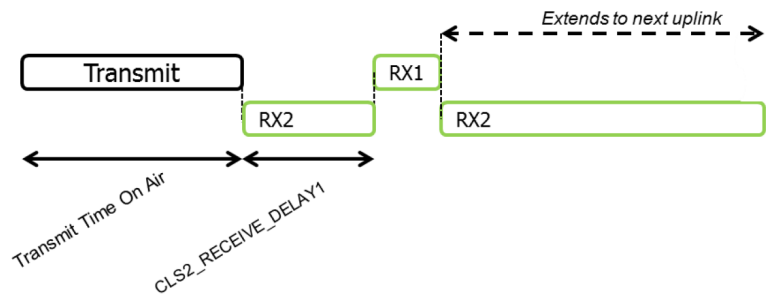

Fig. 23. Class $\mathrm{C}$ end-device reception slot timing

4) Transmission and Retransmission Procedure: It is straightforward in LoRaWAN communication. When an ED has data to transmit, it just wakes up and sends. Class A uses Aloha method, and class B uses slotted Aloha method. Even if the device is moving (location changes), the ED only transmits data, and if any GW in the range receives the message, it will forward the data to NS. Acknowledgement can be used to verify that the message had been received, especially for important data. This is used to avoid data loss when transmitting during mobility. When an ED asks for ACK on the uplink message and ACK is not received, the ED will retransmit the message. In case 1, Figure 6 (a), the ED will retransmit message until:

- Receiving acknowledgment

- Reaching a maximum number of retransmission

It is up to the ED to choose whether to retransmit or forfeit that message and move on.

The ED tries to regain connectivity by lowering the data rate to increase the communication range. While lowest data rate is used, no action can be taken to improve link range. 
In case 2, Figure 6 (b), the NS will retransmit the message until:

- Receiving acknowledgement

- Reaching a maximum number of retransmission (value specified for that ED during configurations)

As for the ED, the NS decides to retransmit or forfeit that message when the ED regains connectivity. As the number of EDs within the range of one GW increases, the uplink frames that require acknowledgements may cause collisions, and the radio network worsens the situation if their responses are not received. This is because EDs use Aloha access method and the retransmission method as explained previously.

Mobility in LoRaWAN can be achieved in uplink, when the device moves, changes the location, and can send data. This can be done using any of the three classes without latency as long as it is under the coverage of LoRaWAN network GWs. Whereas LoRaWAN uses broadcast for uplink, it is more difficult for downlink. The NS selects only one GW to send the message to the ED based on the forwarding table. When the ED moves and changes its location from the coverage of the current GW to another, the NS can no more reach it until a message is received from this device. Considering applications for which latency is constrained, the latency value changes between the three classes. In the downlink, when data are available on NS and ready to send, it will:

- Directly send the data if the ED is operating in class C.

- Send data on one of the pre-defined listen to time slots if the ED is in class B.

- Send data directly when an uplink message is detected from the ED of class A.

\section{B. DASH7 Alliance}

In $\mathrm{D} 7 \mathrm{~A}$, an $\mathrm{ED}$ selects one $\mathrm{GW}$ to communicate. It searches for a $\mathrm{GW}$ within the range and it chooses one to communicate with based on the signal strength. When moving, the ED sends the previously sent data to the same GW. In this case, the ED will not receive any acknowledgement and will detect that the connection is lost due to the location change.

1) Transmission (Uplink) Processes:

In DASH7 the connection is sprightly and straightforward as LoRaWAN. A device uses the CSMA/CA process to transmit a message and will guard the channel before transmission. An ED will communicate with any GW that acknowledges its message. In case the connection is lost with the current $\mathrm{GW}$, the ED will send the next message as broadcast, and it will communicate with the GW that responds to the message. If multiple gateways respond, the ED will choose the best gateway according to link budget (RSS) and start the communication with it in a unicast manner until the connection is lost again. If the ED does not use security, it can communicate with any GW in range. But if network security is implemented, the device can communicate with only the GWs that share the same network key. Network keys are currently pre-shared. The D7A protocol specifications do not include a way or method to assign the keys, but programmers are free to implement their own plan.

\section{2) Reception (Downlink) Processes:}

In the downlink, an ED may receive two types of frames: Background and Foreground frames. Also, there are two ways for communication: Pull and Push. Background frames are sent by the GW to an ED for group synchronization using the pull method. Foreground frames are used for request/response between the ED and GW or two EDs using either push or pull. In the following, we will explain each frame reception process and conclude on how mobility can be achieved.

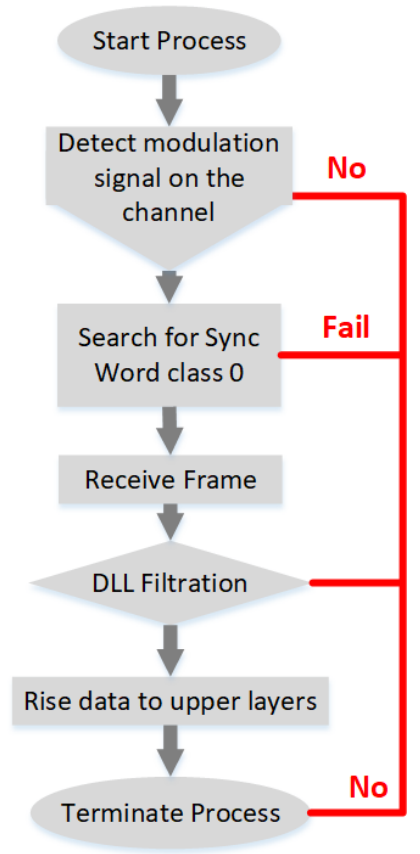

Fig. 24. Background Reception Process

Background Scan and Message Reception: As illustrated in Figure 24, an ED searches periodically for a PHY frame with a Sync Word of class 0 for time $T_{0}$. If successfully received, the ED will decode the received frame. The frame will pass the three filtration steps that DLL supports, that are: subnet, CRC16 and link quality, and ED address if frame is not broadcast. If no frame is received, the process exits immediately. If the frame filtration process is passed successfully, data is transfered to upper layers. Otherwise, the packet is rejected, and the process ends.

Foreground Scan and Message Reception: As illustrated in Figure 25, the ED searches for a PHY frame that has a sync word of class 1 for time $T_{0}$ as explained in the access class. If successfully received, the ED decodes the frame. Then it passes the three filtration steps that DLL supports. When filtration process is successful, the data in the frame transfered to upper layers. If the frame does not pass the filtration, it is rejected. If the value of $T_{o}$ is set to zero, then the process iterates infinitely.

If an ED is static, it is assigned to a specific GW. When there are data to transmit, the ED will send the data to the GW and wait for the response. When moving out of the coverage of the current GW to a new GW coverage, the ED will detect that 


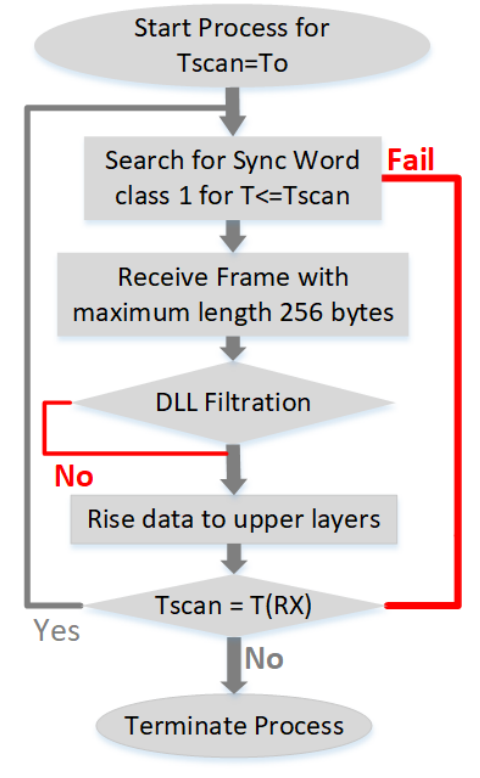

Fig. 25. Foreground Reception Process

the location has changed when no acknowledgement has been received for the transmission. In this case, the ED discovers the new GW in the range using the broadcast message and connect to it. Using the Background scan explained previously, the ED will be updated with the new GW configurations. In the downlink, ED uses the foreground scan to receive the request and data from the GW. Each GW has some EDs assigned to it. When an ED is under the GW coverage, it will respond to the requests attached to it. If there is no response, the GW detects that this ED is no more reachable. Therefore mobility is feasible in D7A.

\section{NB-IoT}

In NB-IoT, an ED connects to only one GW to communicate with, i.e. each ED is associated with a GW. During movement, this ED may change its location several times and, each time the connection is lost, it will search for a suitable GW to connect. When ED has data to transmit (uplink), it will search for a cell on an appropriate frequency, read the SIB information, and will start the random access procedure.

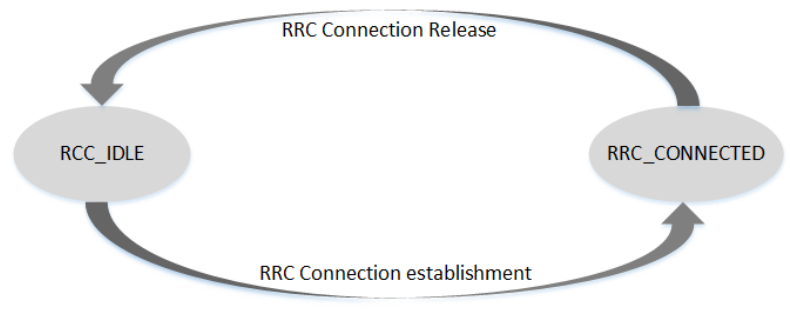

Fig. 26. End-device States in NB-IoT

1) Cell Access: This step is repeated every time an ED looses the connection with the GW. In NB-IoT, an ED has two states as shown in Figure 26, RRC_IDLE (sleeping state) and RRC_CONNECTED (connection state). The handover has been removed because this standard was designed to be simple by reducing the complexity of LTE functions. Communication is considered to be short, with infrequent messages between the ED and the GW, and one GW can serve that. The ED searches for a GW on an appropriate frequency. Then, the connection setup starts as shown in Figure 27. During the connection setup, the ED obtains first the Narrowband physical Cell ID (NCellID) from NSSS channel broadcast by the GW. Second, the ED decodes NCellID to get the NB-MIB, which includes the SIB1-NB size, the number of repetitions, scheduling InfoSIB1 (cell access and selection), and its starting position. Third, the ED decodes SIB1-NB to get the cell access parameters information: PLMNID, TA code, cell identity \& cell status and cell selection information like the minimum receiver level. Fourth, the ED decodes NB-SIB2, that provides it with the configuration information about common logical \& physical channels. Most information in SIB2 is the Random Access Channel (RACH) configuration which is required for uplink synchronization. At this level, the ED initializes and sends the RACH Preamble to the GW. When the GW receives the request, it will respond with $\mathrm{Msg} 2$. If the $\mathrm{GW}$ does not receive the request, the $\mathrm{ED}$ will not receive a response so that it will resend the request. Then, the ED sends Msg3 to start the content resolution process, and the GW sends the response in Msg4 that indicates the successful completion of the RACH procedure. Finally, RRCConnectionRequest suggests that the ED wants to connect to the network.

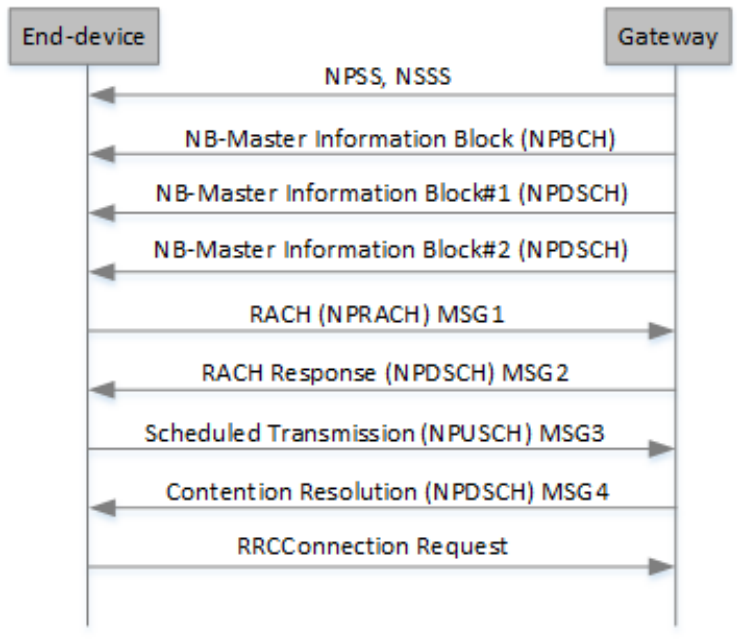

Fig. 27. Connection Setup

2) Mobility: ED may loose the connection when moving far from the GW. So ED changes to RRC_IDLE state to reselect another GW. Setup time is less than 10 s. In [47], results show that setup time is $6.6 \mathrm{~s}$ when NB-IoT is deployed as stand-alone, and about $9.882 \mathrm{~s}$ when it is deployed in-band with LTE and assuming the same results if deployed in guardband. When the GW releases the connection, it sends to the ED the current Access Stratum (AS) contexts to store them. These AS contexts will be used later by the ED to resume 
the connection (faster than cell setup) as shown in Figure 28. Table XVI compares the number of used messages among the three methods available in NB-IoT: legacy service request, RRC connection suspend/resume, and data transmission via the control plane. In resuming process, there are two cases:

- Gateway accepts resume: Switches back to the connection. The cost is five messages.

- Gateway rejects resuming: ED releases stored AS context, returns to IDLE state, then will repeat connection setup. The cost is nine messages.

In uplink, when an ED wakes up, the connection will be resumed if it was established. Otherwise, the ED will search for a GW to connect on. When the connection is established, the ED may transmit data.

In downlink, the GW uses a paging method to trigger an RRC connection which indicates a change in system information for ED in RRC_IDLE mode. It is used for connection setup or system info change. Even if the ED in the RCC_IDLE states is considered sleeping, it still monitors some of the SubFrames (SFs) that are related to paging.

In NB-IoT standard, ED can move between different NBIoT GWs similar to a mobile phone. Even if no handover identical to cellular system handover is supported, but mobility can still be achieved over the X2 interface between two GWs as mentioned previously. When current GW sends the resume connection information to the new GW, ED can resume the connection with the original GW. This method provides ED with fast connection establishment.
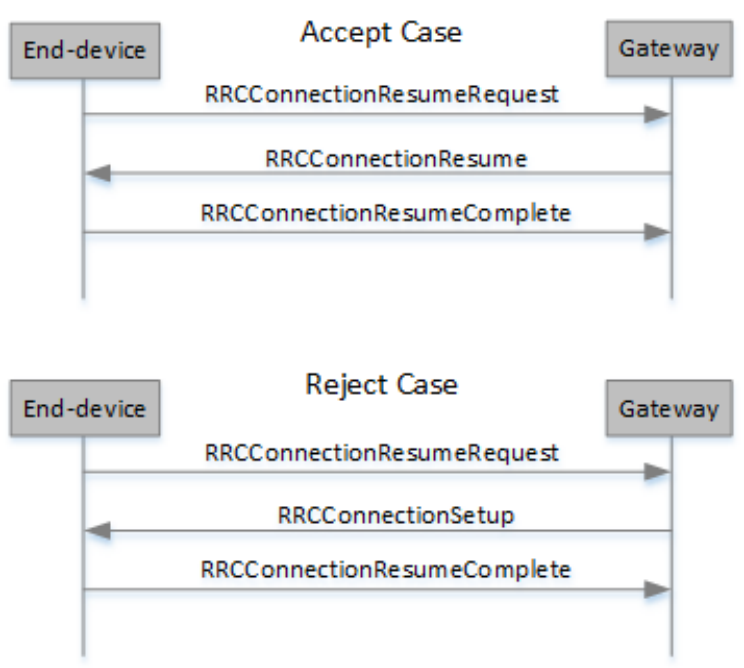

Fig. 28. Connection Resuming Process

\section{COMPaRative STUdy}

The three LPWAN technologies differ in several key features. In the following, we provide a general comparison between the LPWAN technologies and cellular and local area networks. Then, we provide a brief technical comparison between the three LPWANs technologies. In particular, we
TABLE XVI

SIGNALING COMPARISON AMONG DIFFERENT METHODS [45]

\begin{tabular}{|c|c|c|c|}
\hline Direction & $\begin{array}{c}\text { Legacy Service } \\
\text { Request }\end{array}$ & $\begin{array}{c}\text { RRC Connection } \\
\text { Resume }\end{array}$ & $\begin{array}{c}\text { Control } \\
\text { Plane Data } \\
\text { Transmission }\end{array}$ \\
\hline UL & \multicolumn{3}{|c|}{ Preamble } \\
\hline DL & \multicolumn{3}{|c|}{ Random Access Response } \\
\hline $\mathbf{U L}$ & $\begin{array}{c}\text { RRC Connection } \\
\text { Request }\end{array}$ & $\begin{array}{l}\text { RRC Connection } \\
\text { Resume Request }\end{array}$ & $\begin{array}{c}\text { RRC Connection } \\
\text { Request }\end{array}$ \\
\hline DL & $\begin{array}{c}\text { RRC Connection } \\
\text { Setup }\end{array}$ & $\begin{array}{c}\text { RRC Connection } \\
\text { Resume }\end{array}$ & $\begin{array}{c}\text { RRC Connection } \\
\text { Setup }\end{array}$ \\
\hline $\mathbf{U L}$ & $\begin{array}{l}\text { RRC Connection } \\
\text { Setup Complete }\end{array}$ & $\begin{array}{l}\text { RRC Connection } \\
\text { Resume Complete }\end{array}$ & $\begin{array}{l}\text { RRC Connection } \\
\text { Setup Complete }\end{array}$ \\
\hline DL & $\begin{array}{l}\text { Security Mode } \\
\text { Command }\end{array}$ & - & - \\
\hline $\mathbf{U L}$ & $\begin{array}{l}\text { Security Mode } \\
\text { Complete }\end{array}$ & - & - \\
\hline DL & $\begin{array}{l}\text { RRC Connection } \\
\text { Reconfiguration }\end{array}$ & - & - \\
\hline $\mathbf{U L}$ & $\begin{array}{c}\text { RRC Connection } \\
\text { Reconfiguration } \\
\text { Complete }\end{array}$ & - & - \\
\hline $\begin{array}{c}\text { Total } \\
\text { \# of } \\
\text { messages }\end{array}$ & 9 & 5 & 5 \\
\hline
\end{tabular}

consider the following factors: deployment model, cost, network coverage, range, battery life, quality of service, mobility and latency. Finally, we provide a general comparison for the three LPWAN technologies.

\section{A. LPWAN and Cellular Networks}

In the past, most of the applications that require low data rate for a long range were using cellular networks. This type of networks provides the users with many services. Before the emergence of LPWAN technologies, cellular networks had been offering the GSM, GPRS, EDGE, 3G, and 4G technologies. Today, 3G/4G technologies aim to provide users with minimum latency and high data rates for multimedia applications. For this purpose, most of IoT applications were used in the GPRS networks [49-51]. GPRS is a $2.5 \mathrm{G}$ mobile communication that provides a data rate of 56 to $114 \mathrm{kbps}$ with a range up to $26 \mathrm{Km}$. The primary disadvantages of GPRS network are the power consumption and high maintenance cost. Whereas, LPWANs provide long range communication up to $50 \mathrm{Km}$ in rural areas with minimum power consumption (20 dB improvement over GPRS networks), down to onetenth of the energy consumed in GPRS and lower maintenance cost. Moreover, GPRS network capacity is limited to the number of available communication channels, whereas LPWANs technologies optimize the available channels assigned to a particular GW to provide a massive number of EDs under the coverage of one GW. In summary, LPWANs technologies key performance metrics are the energy efficiency, wide coverage and scalability for a low data rate. While cellular networks suffer from high power consumption, complexity, and high deployment cost.

\section{B. LPWAN and Local Area Networks}

From the market perspective, ZigBee and WiFi [52] communications dominate in personal area networks. Each technology 
TABLE XVII

COMPARISON OF THE THREE LPWAN STANDARDS

\begin{tabular}{|c|c|c|c|c|c|c|}
\hline & & \multicolumn{3}{|c|}{ LoRaWAN } & \multirow{2}{*}{ DASH7 } & \multirow{2}{*}{ NB-IoT } \\
\hline & & $\begin{array}{c}\text { Class } \\
\text { A }\end{array}$ & $\begin{array}{c}\text { Class } \\
\text { B }\end{array}$ & $\begin{array}{c}\text { Class } \\
\mathrm{C}\end{array}$ & & \\
\hline \multicolumn{2}{|c|}{ Frequency Band } & \multicolumn{3}{|c|}{$433 / 868 / 780 / 915 \mathrm{MHz}$ ISM } & 433/ 868/915 MHz ISM/SRD & Cellular Band \\
\hline \multicolumn{2}{|c|}{ Channel width } & \multicolumn{3}{|c|}{$500-125 \mathrm{kHz}$} & 25 or $200 \mathrm{kHz}$ & $180 \mathrm{kHz}$ \\
\hline \multicolumn{2}{|c|}{ Spectrum } & \multicolumn{4}{|c|}{ unlicensed } & licensed \\
\hline \multicolumn{2}{|c|}{ Modulation } & \multicolumn{3}{|c|}{ Chirp spread spectrum (CSS) } & GFSK & $\begin{array}{c}\text { DL:QPSK } \\
\text { UL: QPSK (multi-tone) } \\
\pi / 4-\mathrm{QPSK}, \pi / 2 \text {-BPSK (Single-tone) }\end{array}$ \\
\hline \multicolumn{2}{|c|}{ Access Method } & Aloha & Slotted Aloha & Aloha & CSMA/CA & $\begin{array}{l}\text { DL: OFDMA } \\
\text { UL: SCFDMA }\end{array}$ \\
\hline \multicolumn{2}{|c|}{ Data Rate (DL/UL) } & \multicolumn{3}{|c|}{$\begin{array}{l}\text { EU: } 0.3-50 \mathrm{kbps} \\
\text { US: } 0.9-100 \mathrm{kbps}\end{array}$} & $9.6,55.555$ or $166.67 \mathrm{kbps}$ & $\begin{array}{l}\sim 50 \mathrm{kbps} \text { ( DL/ UL multi-tone) } \\
\sim 20 \mathrm{kbps} \text { ( UL single-tone) }\end{array}$ \\
\hline \multicolumn{2}{|c|}{ Duplex } & \multicolumn{3}{|c|}{ Half } & Half & Half \\
\hline \multicolumn{2}{|c|}{ Topology } & \multicolumn{3}{|c|}{ Star } & Star, tree, Node-to-Node & Star \\
\hline \multicolumn{2}{|c|}{ Payload Size } & \multicolumn{3}{|c|}{$51-222$ bytes } & 256 bytes (Max) & $\begin{array}{l}\text { UL: } 125 \text { bytes } \\
\text { DL: } 85 \text { bytes }\end{array}$ \\
\hline \multicolumn{2}{|c|}{ Mobility support } & \multicolumn{3}{|c|}{ High \& Simple } & High \& Simple & High \& Complex \\
\hline \multicolumn{2}{|c|}{ Mobility latency } & \multicolumn{3}{|c|}{ low (Almost Zero) } & low $(305 \mathrm{~ms})[48]$ & High $(1.6-10 \mathrm{~s})$ \\
\hline \multicolumn{2}{|c|}{ Transmission Time } & \multicolumn{3}{|c|}{$\begin{array}{l}\text { Depend on Spreading Factors } \\
\text { Payload size }=10-50 \text { bytes } \\
\mathrm{SF}=7,8,9,10,11==>\mathrm{T}<1 \mathrm{~s} \\
\mathrm{SF}=12==>\mathrm{T}=1-2 \mathrm{~s}\end{array}$} & \multirow{2}{*}{$\begin{array}{l}\text { Advertising: } 1 \mathrm{~s} \\
\text { Request }<50 \mathrm{~ms} \\
\text { Responses: } 1 \mathrm{~s}\end{array}$} & $\begin{array}{l}\text { Depend on block size } \\
\text { ex. } 696 \text { bits }=2.56 \mathrm{~s} \\
\text { and number of repetitions }\end{array}$ \\
\hline \multicolumn{2}{|c|}{ Receiving Time } & $\begin{array}{l}2 \mathrm{~s} \text { if } \\
\mathrm{ON}\end{array}$ & $\begin{array}{l}\text { According } \\
\text { to slot time }\end{array}$ & $\begin{array}{c}\text { Always } \\
\text { unless } \\
\text { Transmitting } \\
\end{array}$ & & $\begin{array}{c}\text { Low } \\
\text { Using Paging Method }\end{array}$ \\
\hline \multicolumn{2}{|c|}{ Transmission Power } & \multicolumn{3}{|c|}{$+14-+27 \mathrm{dBm}$} & $\begin{array}{c}+10(433 \mathrm{MHz}) \\
+27(868 / 915 \mathrm{MHz}) \mathrm{dBm}\end{array}$ & $20 / 23 \mathrm{dBm}$ \\
\hline \multicolumn{2}{|c|}{ DL Latency } & High & Medium & Low & Low & Medium \\
\hline $\begin{array}{r}\text { Suppo } \\
\text { Real-Time ap }\end{array}$ & cations & No & No & Yes & No & No \\
\hline $\begin{array}{l}\text { Device } \\
\text { Access } \mathrm{p}\end{array}$ & & & $\begin{aligned} \mathrm{UL} & >11 \\
\mathrm{DL} & <100\end{aligned}$ & & $\begin{array}{l}\text { NA (connectionless } \\
\text { communication) }\end{array}$ & $\sim 55 \mathrm{k}$ \\
\hline Collisi & & High & Medium & Medium & Low & Low \\
\hline Range (theorti & values) & & $\begin{array}{r}2-5 \mathrm{Km}(\mathrm{u} \\
15 \mathrm{Km}(\mathrm{ru}\end{array}$ & & $\begin{array}{c}1 \mathrm{Km} \text { (node to gateway) } \\
2 \mathrm{Km} \text { (using subcontroller) }\end{array}$ & $\begin{array}{c}\text { Several Km depends on } \\
\text { the number of repetition } \\
10-15 \mathrm{Km} \text { (Rural) }\end{array}$ \\
\hline Link bu & & & up to 157 & & up to $140 \mathrm{~dB}$ & $154 \mathrm{~dB}$ \\
\hline Receiver Se & tivity & & $-124-(-134)$ & & $-97-(-110) \mathrm{dBm}$ & $-141 \mathrm{dBm}$ \\
\hline Multi-hop & port & & No & & Yes (only 2 hops) & No \\
\hline Address & & & $\begin{array}{l}\text { UL: Broad } \\
\text { DL: Unic }\end{array}$ & & $\begin{array}{c}\text { Unicast } \\
\text { Broadcast } \\
\text { Multicast } \\
\text { Anycast }\end{array}$ & $\begin{array}{c}\text { UL: Unicast } \\
\text { DL: Unicast and Broadcast }\end{array}$ \\
\hline Device Addressing & $\begin{array}{c}\text { Fixed } \\
\text { (As MAC) }\end{array}$ & & Unique 64-bit & dress & Unique 64 -bit address & As LTE \\
\hline & $\begin{array}{l}\text { Dynamic } \\
\text { (AS IP) }\end{array}$ & & Unique 32-bit & dress & Unique 16-bit address & \\
\hline Standa & & & LoRa Allia & & DASH7 Alliance & 3gpp (release 2015) \\
\hline Battery & & & & & $\sim 10$ years & \\
\hline
\end{tabular}

offers specific performance and features. ZigBee has been developed and standardized to provide a wireless connection with low power consumption and low data rate (20-250 kbps) for small-scale projects $(10-75 \mathrm{~m})$. ZigBee has been adopted in a wide range of applications, such as medical data collection, home automation, gardening, and other applications that require low power and low data rate. WiFi was designed to provide a high data rate (up to $150 \mathrm{Mbps}$ ) with minimum latency for a local area network (up to $100 \mathrm{~m}$ ). Wifi is efficient for streaming and multimedia data. However, it is a power consumption technology unlike ZigBee and LPWAN technologies. Even though ZigBee is developed and optimized for IoT applications, its prominent problem is limited communication range. Moreover, WiFi and ZigBee use a mesh topology where complexity increases as the number of EDs increase. Mesh topology is used to extend the network communication range especially for short range communication technologies, but it still does not have the long-range capability 
provided by LPWAN technologies. Moreover, mesh is not power efficient especially for battery powered devices, since each ED consumes its battery by repeating the RF signal of the neighbor ED. As number of devices increase, mesh may no more adequately fit the requirements of LPWAN applications. Unlike LPWAN, WiFi and ZigBee do not enable a massive number of wireless connections over an extended range with minimum power consumption since they are limited to small regions [53].

\section{Technical Comparison Between the Three Technologies}

1) Deployment Model and Cost: LoRaWAN, D7A, and NB-IoT operate in the sub-1 GHz bands. LoRaWAN and D7A use unlicensed bands, whereas NB-IoT uses the licensed band. This allows LoRaWAN and D7A to be easily deployed whereas NB-IoT needs to be authorized within the deployment area. NB-IoT devices can benefit from the wide implementation area of the cellular network which can be reused. But the mobility of those devices is limited within the coverage area of a cellular network which is mostly deployed in urban places. Thus in rural or suburban areas where 4G/LTE base stations have not been installed yet, NB-IoT is not suitable. Regarding cost, several aspects should be studied: spectrum cost, network cost, device cost, and deployment cost. In case of LoRa and DASH7, the spectrum is unlicensed whereas NB-IoT spectrum license cost is higher than $\$ 500$ million/ $\mathrm{MHz}$ [54]. Concerning network and deployment cost, LoRa and DASH7 cost between $\$ 100$ - \$1000/gateway whereas that of NB-IoT is $\$ 15000 /$ base station [55]. These values show the advantages for unlicensed bands over licensed ones concerning cost.

2) Network Coverage and Range: In LoRa, one gateway can cover a whole city. The theoretical range [56] of coverage varies between $2-5 \mathrm{~km}$ in urban and up to $15 \mathrm{~km}$ in rural and also depending on the supported ADR method. For example, the LoRaWAN network has been deployed in Belgium, where it covers an entire city with a single gateway only [54]. This is the reason why EDs, in LoRaWAN, transmit data in a broadcast manner without any considerations.

In DASH7, one $\mathrm{GW}$ can cover up to $1 \mathrm{~km}$. This range can be extended to $2 \mathrm{~km}$ if a sub-controller is used. The DASH7 protocol can manage the range of coverage depending on the ADR which is between $28-200 \mathrm{kbps}$ [57]. Unlike in LoRaWAN, an ED in DASH7 is associated with the nearest $\mathrm{GW}$ that is selected based on the best RSS.

In NB-IoT, the range varies between $10-15 \mathrm{~km}$ on rural areas and up to several km's in urban. NB-IoT supports HARQ mechanism to boost the signal power of an ED in extreme coverage conditions. This allows improving the quality of the received signal while operating at low power consumption [58]. Also, NB-IoT benefits from the advantage that cellular networks are already deployed in most cities.

When the overall country is covered with LTE cellular network, using NB-IoT devices will be better than installing LoRa and DASH7 GWs. Otherwise, in rural places where cellular base stations are not present, it is more efficient to install one LoRa GW or several DASH7 GWs than installing LTE base stations for a limited number of EDs.

3) Battery Life and Latency: LoRaWAN features three classes to support several types of applications [59]. Class A offers a low power consumption but with high latency in DL. Class B provides medium latency in DL with a medium power consumption. Class $\mathrm{C}$ provides low latency in DL but with high power consumption. Depending on the application requirements, one of these classes could be applied. Even switching between classes or changing Spreading Factor within the same class is feasible [60]. For example, when an application has to be processed in real-time, an ED can switch to class $\mathrm{C}$ and then return to class $\mathrm{A}$ when the realtime transmission is over.

In D7AP, an ED uses CSMA/CA method to transmit. This method is inappropriate [27] for large/active networks; Latency will increase, as the network expands rapidly. The Device checks available GWs in the range by broadcasting a message. Then the ED selects one GW. This requires more power consumption than class A on LoRaWAN. But D7AP uses ad-hoc synchronization method explained in section II which makes DASH7 more power efficient than NB-IoT.

In NB-IoT, devices consume more power due to the regular synchronization that is not required in Aloha-based systems used by LoRaWAN. Also, OFDM requires more peak current for linear transmitting [61].

DASH7, NB-IoT, and class $\mathrm{C}$ in LoRaWAN represent one of the best choices for applications that require low latency and high data rate. In term of low power consumption, class A \& class B of LoRaWAN would be preferred, followed by DASH7.

4) Message Loss: LoRa and DASH7 standards use unlicensed spectrum's and are asynchronous protocols. The schemes used by DASH7 and LoRa handle interference, fading, and multi-path [62]. Moreover, DASH7 uses three kinds of collision avoidance (AIND, RAIND, and RIGD) [27] but they do not avoid a message loss as NB-IoT. NBIoT is a time slotted synchronous protocol over a licensed spectrum with efficient management of interference. Thus NBIoT uses the HARQ mechanism that increases the message delay to avoid loss of data. In a similar manner, in order to insure data reception, LoRa selects higher spreading factor (SF) which results in low data rate and increased message delay.The results in [38] and [56] show that as the number of devices (up to 250 devices) and packet size ( 50 bytes) increase, the probability of successful transmission decreases (to reach 10\%) in LoRaWAN due to collisions [63]. Applications that require low message loss can select the NB-IoT standard, DASH7 with the AIND method, or LoRa with SF12.

5) Mobility and Latency: In mobility, there are two cases: i. Coverage of two GWs without intersection. ii. Coverage of two GWs with the crossing (intersection). Concerning the first case, it is evident that the ED connects to the GW covering its location. For the second case, we can separately consider the mobility in uplink or downlink. In LoRaWAN, the three classes use broadcast in uplink, so mobility is achieved with 
latency equal to zero. In DASH7, the mobility occurs when no acknowledgement has been received for an uplink with latency equal to $305 \mathrm{~ms}$. Mobility for NB-IoT is similar to that of DASH7 but with a latency up to $9 \mathrm{~s}$. In the downlink, latency in LoRaWAN and DASH7 equals to the time needed for an ED to send an uplink message. Otherwise, they are recognized as unreachable. It is almost the same case for NB-Iot, with a latency equal to the time needed for an ED to send a message in uplink, in addition to the time needed to discover and setup the connection.

\section{LPWANs Technologies: LoRaWAN-DASH7-NB-IoT}

LPWANs technologies share common features: simplicity, star/tree topology (no routing), similar network architecture, sleep mode for maximum power saving, uplink communication, long-range communication, etc. LPWANs do not share the same features with small area network standards. As LPWAN technologies fill the gap between local area networks (Wifi, Bluetooth, ZigBee, etc.) and cellular networks (GSM, 3G, LTE, etc.), DASH7 fills the gap within LPWANs technologies between LoRaWAN and NB-IoT. In LoRaWAN, ED directly sends uplink data to GW without establishing a connection. In LoRaWAN, uplink data are always broadcast. In NB-IoT, ED is assigned to one cell before any uplink message. Connection establishment in NB-IoT requires more than five seconds. The mechanism of communication that ED uses in DASH7 starts by sending the first uplink data in broadcast mode; In this case, it is similar to LoRaWAN ED. Then, based on the received RSSI within the ACK message from one or more GWs, ED selects the GW with the highest RSSI and forwards the following uplink messages; In this case, it is similar to NB-IoT ED. In Downlink, LoRaWAN uses a simple communication scheme. Based on the forward table found in NS, the downlink data is sent to the GW that has been selected according to the highest RSSI. In NB-IoT, GW uses paging to wakeup the ED for downlink or to change its configurations. DASH7 uses a simple synchronization method (D7A Advertisement Protocol) to send a downlink data or to configure the EDs. As for the power consumption, DASH7 consumes less power than NB-IoT and more than LoRaWAN. During mobility, DASH7 requires slight delay compared to LoRaWAN when sending an uplink data and less time than NB-IoT. LoRaWAN time delay is negligible, DASH7 needs $305 \mathrm{~ms}$ to switch between two GWs, while NB-IoT needs at least $5 \mathrm{~s}$ to resume the connection. In term of the QoS, DASH7 provides better quality than LoRaWAN for uplink data but less than NB-IoT.

\section{Application Usage AnALYsis}

The Internet of Mobile Things (IoMT) concept has been widely used recently in applications such as health-care, travel, transportation, etc. In such applications, mobility is one of the major requirements. The IoMT factors and the different features of LoRaWAN, DASH7, and NB-IoT lead to the adequacy of a standard for a given application. From the previous discussions covered in this paper, we conclude that one technology cannot serve all IoMT applications equally. In the following sub-sections, various IoMT application scenarios will be presented. Then, we will discuss how to select the most applicable standard for each scenario. In general, the three standards can serve two user groups: businesses and individuals as mentioned in the following:

- Use-cases for end-customers:

- Wearables: These devices measure, monitor and analyze the human daily activities (e.g. smart watches, etc.).

- Connected Cars: Cars connect to Internet using certain technology. This type of devices provides the driver with safety and information about the driving environment.

- Personal Health: Beyond fitness tracking wearables, these devices have countless pathological and therapeutic uses.

- Use-cases for businesses:

- Health care: They are used to take care of patients in real time. Hospitals use IoT to track the location of medical devices, individuals and patients who can extend preventive care outside the hospital premises.

- Smart Cities: Large-scale connectivity, big data, analysis and financing are being transformed from smart city initiatives and public services such as waste management, traffic management, water distribution, urban safety and environmental monitoring.

\section{A. Pallet Tracking for Logistics}

Today, transportation platforms for the location and condition of goods are required [64]. In such applications, the most frequently requested requirements are a device with low cost, small size, easily deployed and powered by a long-life battery. Pallet tracking can be a good scenario for mixed distribution solutions. Logistics companies may own their networks in their facilities to provide guaranteed coverage. Public GWs supplied by LoRaWAN, DASH7 or LTE base station supporting NB-IoT can be used when vehicles are out of company coverage or when goods arrive at customers' premises. During fast motion in rural areas, LoRaWAN enables more reliable connections than DASH7. In urban areas, DASH7 offers more reliability and provides an accurate location within the coverage of the GW. For NB-IoT, LTE may not be available at all logistics sites, especially in rural areas. In this scenario, the requirements are low cost, extended life battery, uplink communication, reliable mobile communications, and long range coverage. LoRaWAN class A can be best suited for this application.

\section{B. Health-care}

One of the most attractive applications in IoT is the medical and healthcare [16], [17], [20], [21]. Usually, when we address this subject, readers think of the wearable devices that are used to remotely monitor the health of the patient, fitness programs, elderly care, and trace a chronic disease. Wearable 
devices, LPWANs, medical servers, and health databases play vital roles in establishing health records and providing health services upon request to accredited stakeholders. During monitoring and evaluation, the patient may move while walking, by car or by train. The requirements for such devices are medium power consumption (usually rechargeable), low cost, mostly uplink rarely downlink communication, reliable mobile communications, QoS, and extended coverage. When moving at high speed (e.g. inside a car or train), DASH7 is preferable to guarantee that the message reaches its destination. LoRaWAN class $\mathrm{C}$ can be used in the absence of DASH7. At low-speed movement, NB-IoT is the best choice especially in urban areas. This allows providing the application with better QoS and reducing data loss. NB-IoT is not efficient at high speeds since it requires up to 10 seconds to establish a connection when switching between two gateways.

\section{Wildlife Tracking and Monitoring}

More than 400 species of animals are threatened with extinction in Africa and in different nature reserves. Monitoring is essential for tracking the patterns of movement of animals and the demographic composition of the population, providing early warning of societal hazards such as bioterrorism, habitat use, monitoring of natural environmental hazards, human health, conservation of endangered species and illegal fishing incidents and breakages [65]. Animals are the perfect observation tools for humans because they share the same environment as humans and spend more time outdoors than humans, which increases the risk of exposure. The IoT can be used to gather valuable information in daily activities to track and control endangered species. Animals are an excellent channel for monitoring new pathogens known as outbreak potentials, given that more than $60 \%$ of the emerging infectious diseases in humans originated as zoonotic diseases [65]. In such remote areas (e.g. Africa and nature reserves) cellular signal/coverage does not exist with high difficulty to deploy. For that, NB-IoT technology is not preferred. For such application, the requirements of the device are low power consumption, low cost, uplink and downlink communication, a device to device communication, and long-range coverage. DASH7 will be best suited for such situation. DASH7 features a tag-to-tag (M2M) communication without the need for network coverage. DASH7 supports a communication for a distance that extends up to $1 \mathrm{Km}$. With DASH7, the scientist can specify the location of the animal as well as its conditions. Moreover, this type of communication does not generate any additional cost, such as device subscription as in LoRaWAN and NB-IoT. LoRaWAN class A can be applied if network coverage exists.

\section{Agriculture and Smart Farming}

Recently, IoT has been adopted and deployed in the agriculture sector. A massive number of sensors is deployed to provide farmers with accurate measurements of grown seeds, soil water and nutrients, amount of used fertilizer, and the temperature of the stored product. IoT has contributed to the doubling and improvement of production [66]. In remote areas (e.g. farms) cellular signal/coverage is either weak or not available and the deployment of such technology is costly. For that, NB-IoT technology is not preferred. The usage of tractors, vehicles, human, and recently the drones and robotics makes up from the mobility parameter a major requirement in agricultural IoT [66], [67]. Usually, agricultural applications require essentially uplink communication for a long distance and low power consumption. Thus, LoRaWAN class A is most preferable in such situation. While DASH7 is not preferable as the communication range increases above $2 \mathrm{Km}$. In this domain, many companies are involved in enhancing the agriculture sector by adopting IoT, like Microsoft in the project FarmBeats [66], Climate Corp, AT\&T, and Monsanto.

\section{E. Smart Vehicles}

In the recent years, the number of cars connected to the Internet has been increasing [68]. The automotive network paved the way for a new category of advanced applications ranging from automotive surveillance and diagnostics to passenger assistance such as road navigation, weather maps and automatic royalty payments, as well as communications and entertainment. Moreover, M2M communication reduces the accidents and assists in routing vehicles to reduce road jams. In such situation, mobility and downlink communication are critical requirements. NB-IoT can be best suited especially in the cities where the number of downlink messages increases due to traffic jam, accidents, road reparation, broken down cars, etc. LoRaWAN is not favored, since it is designed for mostly uplink communication. DASH7 is an excellent choice within the cities. DASH7 features the M2M communication that offers communication between vehicles directly without passing by the network.

\section{CONCLUSION}

This paper provided an overview of the three low power wide area networks: LoRaWAN, DASH7 Alliance, and Narrow-Band IoT. The architecture of each standard has been explained, and the mobility mechanisms were reported. Mobility management within the same standard/technology was also introduced. Then, a comparative study of the three standards was presented including a shortened table that contains most of the essential parameters for each standard. This review addressed the mobility for ED within the same standard. For each standard, the mobility management is explained and puts forward either the simplicity or difficulty. Future works will consider the mobility management of ED between different standards under heterogeneous technologies. Besides the three standards illustrated in this paper, and within the LPWANs technologies set, other standards can be used, like Sigfox, LTE-M, Wi-SUN, etc. We will focus on a new set of LPWANs standards in a future study following the same methodology.

\section{REFERENCES}

[1] U. Raza, P. Kulkarni, and M. Sooriyabandara, "Low power wide area networks: An overview," IEEE Communications Surveys Tutorials, vol. 19, pp. 855-873, Secondquarter 2017. 
[2] "Introducing new strategy to unify iot services under smartthings, in addition to the next-generation bixby 2.0, and advanced ar capabilities on more galaxy smartphones." Samsung Developer Conference, October 18, 2017, https://www.sdc2017.com.

[3] Vodafone, "Benchmarks and best practices to help achieve the greatest return from internet of things projects." White Paper [Online], https://www.vodafone.com/iot, Sep 2017.

[4] J. Wu, S. Guo, H. Huang, W. Liu, and Y. Xiang, "Information and communications technologies for sustainable development goals: Stateof-the-art, needs and perspectives," IEEE Communications Surveys Tutorials, vol. PP, no. 99, pp. 1-1, 2018.

[5] D. Evans, "The internet of things: How the next evolution of the internet is changing everything." CISCO white paper, vol. 1, 2011.

[6] L. Z. Shelby, "Embedded web services." IEEE Wireless Communications [Online], vol. 17, no.6, pp. 52-57, Dec. 2010.

[7] "Gartner says 6.4 billion connected things will be in use in 2016, up 30 percent from 2015," November 10, 2015.

[8] J. Wu, S. Guo, J. Li, and D. Zeng, "Big data meet green challenges: Big data toward green applications," IEEE Systems Journal, vol. 10, pp. 888-900, Sept 2016.

[9] K. Wang, Y. Wang, Y. Sun, S. Guo, and J. Wu, "Green industrial internet of things architecture: An energy-efficient perspective," IEEE Communications Magazine, vol. 54, pp. 48-54, December 2016.

[10] R. Atat, L. Liu, H. Chen, J. Wu, H. Li, and Y. Yi, "Enabling cyberphysical communication in $5 \mathrm{~g}$ cellular networks: challenges, spatial spectrum sensing, and cyber-security," IET Cyber-Physical Systems: Theory Applications, vol. 2, no. 1, pp. 49-54, 2017.

[11] C. Perera, C. H. Liu, and S. Jayawardena, "The emerging internet of things marketplace from an industrial perspective: A survey," IEEE Transactions on Emerging Topics in Computing, vol. 3, pp. 585-598, Dec 2015

[12] J. Wu, S. Guo, J. Li, and D. Zeng, "Big data meet green challenges: Greening big data," IEEE Systems Journal, vol. 10, pp. 873-887, Sept 2016.

[13] P. Patel, M. I. Ali, and A. Sheth, "On using the intelligent edge for iot analytics," IEEE Intelligent Systems, vol. 32, pp. 64-69, September 2017.

[14] R. Morello, S. C. Mukhopadhyay, Z. Liu, D. Slomovitz, and S. R Samantaray, "Advances on sensing technologies for smart cities and power grids: A review," IEEE Sensors Journal, vol. 17, pp. 7596-7610, Dec 2017

[15] A. Zanella, N. Bui, A. Castellani, L. Vangelista, and M. Zorzi, "Internet of things for smart cities," IEEE Internet of Things Journal, vol. 1, pp. 22-32, Feb 2014.

[16] G. Z. Papadopoulos, V. Kotsiou, A. Gallais, G. Oikonomou, P. Chatzimisios, T. Tryfonas, and T. Noel, "A mobility-supporting mac scheme for bursty traffic in iot and wsns," in 2016 IEEE Global Communications Conference (GLOBECOM), pp. 1-6, Dec 2016.

[17] S. M. R. Islam, D. Kwak, M. H. Kabir, M. Hossain, and K. S. Kwak, "The internet of things for health care: A comprehensive survey," IEEE Access, vol. 3, pp. 678-708, 2015.

[18] P. Hank, S. Mller, O. Vermesan, and J. V. D. Keybus, "Automotive ethernet: In-vehicle networking and smart mobility," in 2013 Design, Automation Test in Europe Conference Exhibition (DATE), pp. 17351739, March 2013.

[19] Y. Shibata and G. Sato, "Iot based mobility information infrastructure in challenged network environment toward aging society," in 201731 st International Conference on Advanced Information Networking and Applications Workshops (WAINA), pp. 645-648, March 2017.

[20] A. J. Jara, D. Fernandez, P. Lopez, M. A. Zamora, and A. F. Skarmeta, "Lightweight mobile ipv6: A mobility protocol for enabling transparent ipv6 mobility in the internet of things," in 2013 IEEE Global Communications Conference (GLOBECOM), pp. 2791-2797, Dec 2013.

[21] M. S. Shahamabadi, B. B. M. Ali, P. Varahram, and A. J. Jara, "A network mobility solution based on 6lowpan hospital wireless sensor network (nemo-hwsn)," in 2013 Seventh International Conference on Innovative Mobile and Internet Services in Ubiquitous Computing, pp. 433-438, July 2013.

[22] N. A. Surobhi and A. Jamalipour, "An iot-based middleware for mobility management in post-emergency networks," in 2014 21st International Conference on Telecommunications (ICT), pp. 283-287, May 2014.

[23] W. Dargie and J. Wen, "A seamless handover for wsn using lms filter," in 39th Annual IEEE Conference on Local Computer Networks, pp. 442445, Sept 2014.
[24] K. Nahrstedt, H. Li, P. Nguyen, S. Chang, and L. Vu, "Internet of mobile things: Mobility-driven challenges, designs and implementations," in 2016 IEEE First International Conference on Internet-of-Things Design and Implementation (IoTDI), pp. 25-36, April 2016.

[25] M. Mohammadkarimi, M. A. Raza, and O. A. Dobre, "Signature-based nonorthogonal massive multiple access for future wireless networks: Uplink massive connectivity for machine-type communications," IEEE Vehicular Technology Magazine, 2018.

[26] LoRa Alliance, https://www.lora-alliance.org/lorawan-for-developers, LoRaWAN Specification, 2016 ed.

[27] H. Lee, S. H. Chung, Y. S. Lee, and Y. Ha, "Performance comparison of dash7 and iso/iec 18000-7 for fast tag collection with an enhanced csma/ca protocol," in 2013 IEEE 10th International Conference on High Performance Computing and Communications 2013 IEEE International Conference on Embedded and Ubiquitous Computing, pp. 769-776, Nov 2013.

[28] H. Lehpamer, "Rfid design principles." Artech House, 2012.

[29] DASH7 Alliance Std., "http://www.dash7-alliance.org/dash7-allianceprotocol-specification-v1-1-ready-for-download/', DASH7 Alliance Mode Specification, 2017 ed.

[30] R. Ratasuk, N. Mangalvedhe, and A. Ghosh, "Overview of lte enhancements for cellular iot," in 2015 IEEE 26th Annual International Symposium on Personal, Indoor, and Mobile Radio Communications (PIMRC), pp. 2293-2297, Aug 2015.

[31] A. Daz-Zayas, C. A. Garca-Prez, . M. Recio-Prez, and P. Merino, "3gpp standards to deliver lte connectivity for iot," in 2016 IEEE First International Conference on Internet-of-Things Design and Implementation (IoTDI), pp. 283-288, April 2016.

[32] R. Ratasuk, A. Prasad, Z. Li, A. Ghosh, and M. A. Uusitalo, "Recent advancements in $\mathrm{m} 2 \mathrm{~m}$ communications in $4 \mathrm{~g}$ networks and evolution towards 5g," in 2015 18th International Conference on Intelligence in Next Generation Networks, pp. 52-57, Feb 2015.

[33] G. T. 36.888, "Study on provision of low-cost machine-type communications (mtc) user equipments (ues) based on lte." v.12.0.0, June 2013

[34] G. T. 36.888, "Cellular system support for ultra low complexity and low throughput internet of things." Nov. 2017. [Online]. Available: http://www.3gpp.org/ftp/Specs/archive/45_series/45.820/45820-d10.zip.

[35] Ericsson and N. Networks, "Further lte physical layer enhancements for mtc.” RP-141660, 3GPP TSG RAN Meeting \#65, Sept. 2017. [Online]. Available: http://www.3gpp.org/ftp/tsg_ran/tsg_ran/TSGR_65/Docs/RP141660.zip.

[36] 3rd Generation Partnership Project (3gpp), http://www.3gpp.org/ftp/Specs/archive/45_series/45.820/, Cellular system support for ultra-low complexity and low throughput Internet of Things (CIoT) (Release 13), 2015 ed.

[37] F. Sforza, "Communications system," Mar. 26 2013. US Patent 8,406,275, https://www.google.com/patents/US8406275.

[38] F. Adelantado, X. Vilajosana, P. Tuset-Peiro, B. Martinez, J. MeliaSegui, and T. Watteyne, "Understanding the limits of lorawan," IEEE Communications Magazine, vol. 55, no. 9, pp. 34-40, 2017.

[39] "Semtech homepage, http://www.semtech.com/," 2017.

[40] LoRa Alliance, https://www.lora-alliance.org/lorawan-for-developers, LoRaWAN Regional Parameters, 2016 ed.

[41] M. Weyn, G. Ergeerts, L. Wante, C. Vercauteren, and P. Hellinckx, "Survey of the dash7 alliance protocol for $433 \mathrm{mhz}$ wireless sensor communication," International Journal of Distributed Sensor Networks, vol. 9, no. 12, p. 870430, 2013.

[42] M. Weyn, G. Ergeerts, R. Berkvens, B. Wojciechowski, and Y. Tabakov, "Dash7 alliance protocol 1.0: Low-power, mid-range sensor and actuator communication," in 2015 IEEE Conference on Standards for Communications and Networking (CSCN), pp. 54-59, Oct 2015.

[43] "Nb-iot deployment, https://www.ericsson.com/research-blog/wpcontent/uploads/2016/05/fig-2.png," 2017.

[44] J. Schlienz and D. Raddino, "NarrowBand Internet of Things," Tech. Rep. 1MA266_0e, Rohde and Schwarz, https://cdn.rohdeschwarz.com/pws/dl_downloads/dl_application/application_notes /1ma266/1MA266_0e_NB_IoT.pdf, 122016.

[45] R. Ratasuk, N. Mangalvedhe, Y. Zhang, M. Robert, and J. P. Koskinen, "Overview of narrowband iot in lte rel-13," in 2016 IEEE Conference on Standards for Communications and Networking (CSCN), pp. 1-7, Oct 2016.

[46] S. Imadali, A. Kaiser, F. Sivrikaya, N. E. Sayed, M. Boc, W. Klaudel, A. Petrescu, and V. Veque, "A review of network mobility protocols 
for fully electrical vehicles services," IEEE Intelligent Transportation Systems Magazine, vol. 6, pp. 80-95, Fall 2014.

[47] R. Ratasuk, B. Vejlgaard, N. Mangalvedhe, and A. Ghosh, "Nb-iot system for $\mathrm{m} 2 \mathrm{~m}$ communication," in 2016 IEEE Wireless Communications and Networking Conference, pp. 1-5, April 2016.

[48] W. Ayoub, F. Nouvel, A. E. Samhat, J. Prevotet, and M. Mroue, "Overview and measurement of mobility in dash7," in 2018 25th International Conference on Telecommunications (ICT), pp. 532-536, June 2018.

[49] H.-W. Ferng and Y.-C. Tsai, "Using priority, buffering, threshold control, and reservation techniques to improve channel-allocation schemes for the gprs system," IEEE Transactions on Vehicular Technology, vol. 54, pp. 286-306, Jan 2005.

[50] A. Damnjanovic, J. Montojo, Y. Wei, T. Ji, T. Luo, M. Vajapeyam, T. Yoo, O. Song, and D. Malladi, "A survey on 3gpp heterogeneous networks," IEEE Wireless Communications, vol. 18, pp. 10-21, June 2011.

[51] N. Xia, H. H. Chen, and C. S. Yang, "Radio resource management in machine-to-machine communications x2014; a survey," IEEE Communications Surveys Tutorials, vol. 20, pp. 791-828, Firstquarter 2018.

[52] A. Aragon-Zavala, Indoor Wireless Technologies, pp. 440-. Wiley Telecom, 2017.

[53] J. S. Lee, Y. W. Su, and C. C. Shen, "A comparative study of wireless protocols: Bluetooth, uwb, zigbee, and wi-fi," in IECON 2007 - 33rd Annual Conference of the IEEE Industrial Electronics Society, pp. 4651, Nov 2007.

[54] LoRa-Alliance, "Lorawan what is it," Tech. Rep. Technical Marketing Workgroup 1.0, https://www.loraalliance.org/portals/0/documents/whitepapers/LoRaWAN101.pdf., November 2016.

[55] R. S. Sinha, Y. Wei, and S.-H. Hwang, "A survey on lpwa technology: Lora and nb-iot," ICT Express, vol. 3, no. 1, pp. 14 - 21, 2017

[56] N. Vatcharatiansakul, P. Tuwanut, and C. Pornavalai, "Experimental performance evaluation of lorawan: A case study in bangkok," in 2017 14th International Joint Conference on Computer Science and Software Engineering (JCSSE), pp. 1-4, July 2017.

[57] O. Cetinkaya and O. B. Akan, "A dash7-based power metering system," in 2015 12th Annual IEEE Consumer Communications and Networking Conference (CCNC), pp. 406-411, Jan 2015.
[58] Y. D. Beyene, R. Jantti, K. Ruttik, and S. Iraji, "On the performance of narrow-band internet of things (nb-iot)," in 2017 IEEE Wireless Communications and Networking Conference (WCNC), pp. 1-6, March 2017.

[59] P. S. Cheong, J. Bergs, C. Hawinkel, and J. Famaey, "Comparison of lorawan classes and their power consumption," in 2017 IEEE Symposium on Communications and Vehicular Technology (SCVT), pp. 1-6, Nov 2017.

[60] T. Petri, M. Goessens, L. Nuaymi, L. Toutain, and A. Pelov, "Measurements, performance and analysis of lora fabian, a real-world implementation of lpwan," in 2016 IEEE 27th Annual International Symposium on Personal, Indoor, and Mobile Radio Communications (PIMRC), pp. 1-7, Sept 2016.

[61] C. Jiao, Z. Zhang, and C. Zhong, "Impact of mobility on the sum rate of an nb-ofdma based mobile iot networks," in 2016 IEEE International Conference on Communications (ICC), pp. 1-6, May 2016.

[62] D. Magrin, "Network level performances of lora system," Dec. 142016. Access in November 2017, http://tesi.cab.unipd.it/53740/

[63] D. Magrin, M. Centenaro, and L. Vangelista, "Performance evaluation of lora networks in a smart city scenario," in 2017 IEEE International Conference on Communications (ICC), pp. 1-7, May 2017.

[64] DHL-Trend-Research and Cisco-Consulting-Services, "Internet of things in logistics," Tech. Rep. Technical Marketing Workgroup 1.0, A collaborative report by DHL and Cisco on implications and use cases for the logistics industry, 2015.

[65] J. P. S. Neo and B. H. Tan, "The use of animals as a surveillance tool for monitoring environmental health hazards, human health hazards and bioterrorism," Veterinary Microbiology, vol. 203, pp. 40 - 48, 2017.

[66] D. Vasisht, Z. Kapetanovic, J.-h. Won, X. Jin, R. Chandra, A. Kapoor, S. N. Sinha, M. Sudarshan, and S. Stratman, "Farmbeats: An iot platform for data-driven agriculture," in Proceedings of the 14th USENIX Conference on Networked Systems Design and Implementation, NSDI'17, (Berkeley, CA, USA), pp. 515-528, USENIX Association, 2017.

[67] D. Floreano and R. J. Wood, "Science, technology and the future of small autonomous drones," Nature, vol. 521, pp. 460-466, 2015.

[68] Z. Zhu, L. Zhang, and R. Wakikawa, "Supporting mobility for internet cars," IEEE Communications Magazine, vol. 49, pp. 180-186, May 2011. 